\title{
A Nonlinear Transfer Function Based Receiver for Wideband Interference Suppression
}

\author{
Kuangyuan Ying, Hao Gao, Dusan Milosevic, and Peter Baltus \\ Mixed-Signal Microelectronics Group, Electrical Engineering Department, \\ Eindhoven University of Technology, Eindhoven, Netherlands \\ Correspondence should be addressed to Kuangyuan Ying; k.ying@tue.nl, Hao Gao; h.gao@tue.nl, \\ and Dusan Milosevic; d.milosevic@tue.nl
}

Received 7 October 2016; Revised 15 December 2016; Accepted 20 December 2016; Published 31 January 2017

Academic Editor: Stephane Evoy

Copyright (C) 2017 Kuangyuan Ying et al. This is an open access article distributed under the Creative Commons Attribution License, which permits unrestricted use, distribution, and reproduction in any medium, provided the original work is properly cited.

Wideband receivers for multistandards operation can simplify the system and lower the cost. In a wideband receiver, the tolerance of large interference signal within the operating band is important. Traditional frequency-domain filtering suffers from lacking in filtering capability for in-band interference signals. This paper describes a receiver system exploiting nonlinear transfer function. Based on the fundamental nonlinear theory, the receiver with nonlinear method can provide frequency-independent filtering for large blockers and linear amplification for weak desired signals simultaneously. The interference suppression performance depends on the amplitude discrimination between the envelope of the large and small signal. The operation of the nonlinear receiver is based on the amplitude of the interferer envelope. A feedforward path is designed to extract the envelope information of the interferer and a feedback path is added to keep track of the environment. With frequency-independent filtering, the nonlinear receiver system enhances both in-band and out-of-band linearity, thus enabling wideband multimode operation.

\section{Introduction}

Wireless communication systems are developing to provide higher speed with reliability under the increasing amount of daily usage [1]. In a mobile handset device, several wireless communication standards are supported, such as 2G/3G/4G, Bluetooth, WiFi, GPS, and NFC [2]. In general, there are two solutions for multiple standards operation. One is narrowband solution and another is wideband solution. In the narrowband solution, multiple narrowband receiver front-ends and off-chip surface-acoustic-wave (SAW) filters are required. In the wideband solution, a single wideband receiver covers the spectrum of interest. The wideband solution is widely applied in the design of software-defined radios (SDR) [3-8] and reconfigurable receivers [9-12]. However, the wideband operation introduces wideband interference problems. The interference comes from the simultaneous operation of multiple radios with multiple standards. The transmitted signal generates interference through the poor isolation between transmitter and receiver in the same device. Also, the transmitted signal generates interference for other devices if they have active receivers operating at the same time.

The multiradio coexistence scenario $[13,14]$ is shown in Figure 1. In Figure 1, there are three wireless terminals. Terminal \#1 is a multiradio device. Terminal \#M and terminal \#N are single-radio devices. Terminal \#1 has both a receiver and a transmitter. The receiver in terminal \#1 is receiving information with wireless standard $\mathrm{A}$, while the transmitter in terminal \#1 is transmitting with wireless standard B. Terminal \#M and terminal \#N are transmitting information through wireless standards $\mathrm{M}$ and $\mathrm{N}$, respectively. In this case, the receiver in terminal \#1 is plagued by the transmitting signal from either the transmitter in terminal \#1, terminal \#M, or terminal \#N. In this scenario, the term "victim" is used for the receiver in terminal \#1, and the term "aggressor" for transmitters in terminal \#1, terminal \#M, and terminal \#N. The input spectrum at the receiver antenna in terminal \#1 is shown accordingly. Due to the difference of wireless standards and coupling paths, the interference signals received at the receiver antenna are different in frequencies and power. The interference signal from the collocated transmitter in the 


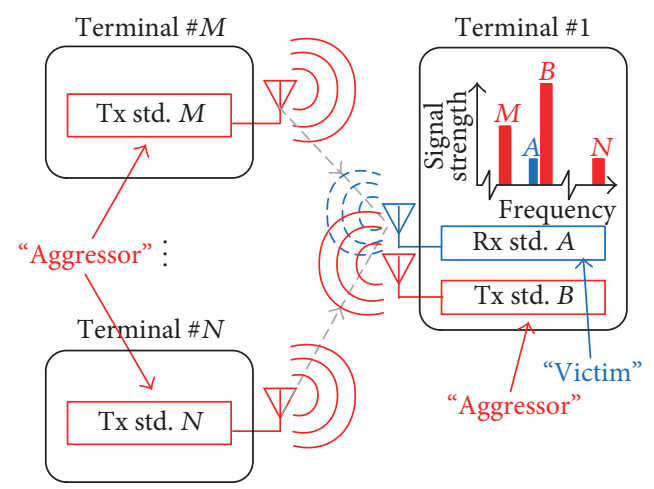

Figure 1: Multiradio coexistence scenario. The receiver of standard A in terminal \#1 is plagued by the strong signal transmitting by either terminal \#1, terminal \#M, or terminal \#N.

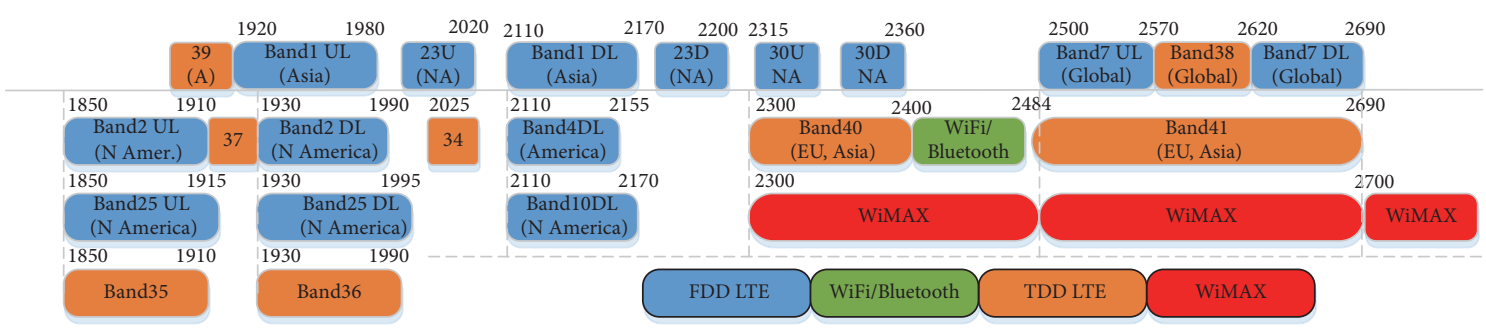

FIgURE 2: Frequency allocation of different wireless standards from $1800 \mathrm{MHz}$ to $2700 \mathrm{MHz}$.

same device (terminal \#1) is usually much stronger because of the small size of a handset device.

The lack of RF filtering after antenna generates problems for wideband operation. The problems can be divided into three categories: distortion, phase noise, and power consumption. It can be extremely harmful if the strong interferer is located close to the desired signal or at harmonic frequency of the desired signal. Firstly, if the interferer is too large, it leads to desensitization of the receiver. Secondly, when the interferer mixes with LO phase noise, it poses additional noise in the receiver band. That noise is proportional to the interferer power $[7,8]$. The receiver's blocker NF under reciprocal mixing can be expressed as

$$
\begin{aligned}
\mathrm{NF}_{\text {blocker }} \approx & -174[\mathrm{dBm} / \mathrm{Hz}]+P_{b}[\mathrm{dBm}] \\
& +L_{\omega}\{\Delta \omega\}[\mathrm{dBc} / \mathrm{Hz}]
\end{aligned}
$$

where $L_{\omega}\{\Delta \omega\}$ is the LO phase noise at the interferer offset $\Delta \omega$ and $P_{b}$ is the interferer power. Thirdly, in order to handle a large interferer, the linear receiver should have a large dynamic range, which would increase the receiver's power consumption. However, if some interference suppression can be provided at the RF stage, all three problems are alleviated.

This paper presents a nonlinear receiver topology with frequency-independent interference tolerance. Based on the information of envelope amplitude of the interferer, the receiver is able to provide suppression at RF frequency for large interferers. It can achieve both good IB and OOB linearity, thus making it suitable for the multiradio coexistence scenario. The suppression at RF frequency also alleviates the requirements for the following receiver circuit blocks and saves the overall power consumption. The paper is organized as follows. Section 2 classifies different interference cases and reviews prior works. The fundamental theory of the nonlinear concept is presented in Section 3. The system modeling and analysis of nonlinear interference suppression for local interference are carried out in Section 4. Section 5 discusses the nonlinear interference suppression for general interference by extracting envelope information. The nonlinear receiver system operation under multiple large interferers is discussed in Section 6. Conclusions are drawn in Section 7.

\section{Interference Cases and Review of Prior Works}

2.1. Interference Scenarios and Cases. There are several wireless standards operating in a mobile handset device. Figure 2 illustrates the frequency allocation of different standards supported in mobile devices, starting from $1800 \mathrm{MHz}$ to $2700 \mathrm{MHz}$. In this frequency range, there are several dominant communication standards, which are FDD LTE, TDD LTE, WiFi, Bluetooth, and WiMAX, and so forth. The frequency spectrum is allocated differently for different countries and regions [1, 15-17]. Several key points are observed here. Firstly, the smallest frequency separation between uplink and downlink of FDD LTE is $20 \mathrm{MHz}$. Secondly, the smallest frequency separation between $\mathrm{WiFi}$ and LTE is $12 \mathrm{MHz}$. Thirdly, FDD LTE, TDD LTE, WiFi, Bluetooth, and WiMAX share a large frequency spectrum. Therefore, the multiradio coexistence in this frequency range requires filtering for interferers located very close to the desired signal in frequency domain. 


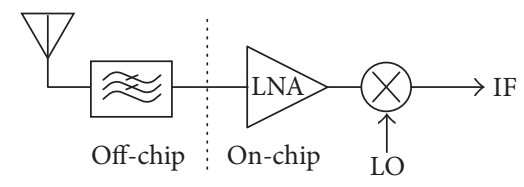

FIGURE 3: Simplified system architecture of a conventional narrowband receiver.

The interference scenarios can be divided into two classes in terms of physical distance, namely, collocation and proximity. The collocation scenario refers to that multiple radios are placed in the same physical unit that the interferers are generated locally inside the device. The transmitting power of LTE user equipment is $24 \mathrm{dBm}$. The measured antenna coupling for collocated $915 \mathrm{MHz}$ patch antennas is roughly $20 \mathrm{~dB}$ in worst case [13].

The proximity scenario happens when multiple devices are placed very close. The transmitting signal from device \#A generates interference for receivers in other devices. Therefore, the interferers are generated externally, for example, from the use of LTE small cell access points and low-power $\mathrm{WiFi}$ routers [18]. The typical transmit power of an access point is around 20 to $30 \mathrm{dBm}$. The free space loss at $2.4 \mathrm{GHz}$ for a distance of $0.5 \mathrm{~m}$ is $34 \mathrm{~dB}$. Therefore, for both scenarios, the interference power can as strong as several $\mathrm{dBm}$.

The interference scenarios of interest can be summarized into three cases.

(1) Out-Of-Band Interferers. Interferers are using different standards and far away in frequency.

(2) Interferers from Other Standards and Close in Frequency (Tens of $\mathrm{MHz}$ ), for Example, WiFi and LTE Coexistence. This case includes interferers generated locally in the same device due to antenna coupling and externally from other devices. Both interferers' power can be several $\mathrm{dBm}$.

(3) Interferers from the Same Standard, In-Band (IB) Interference, for Example, WiFi/WiFi, LTE/LTE, and FDD LTE $U P / D N$. This case includes interferers generated locally due to TX leakage of FDD/FD systems and externally from other devices. TX leakage on RX depends on the isolation provided by the duplexer. Both interferers' power can be several $\mathrm{dBm}$.

2.2. Interference Cancelling/Filtering Methods. In a conventional narrowband receiver as shown in Figure 3, RF signals and interference are bandpass-filtered by an off-chip SAW filter after the receiver antenna. In this way, the unwanted OOB interferers are filtered out. When the SAW-based filters are omitted after the receiver antenna, the receiver needs to provide blocker filtering and harmonic rejection function to meet the target of wireless communication specifications.

In FDD communication systems shown in Figure 4(a), a duplexer [19] is placed after the single antenna to allow bidirectional communication of receiving and transmitting signals. The functions of the duplexer are to provide matching band selection and to attenuate the transmitter leakage at the receiver input for avoiding desensitization of the receiver. The duplexer relies on frequency-selective filters for isolation and band selection, which often means high-Q and off-chip.

Figure 4(b) shows an analog cancellation technique [20] for locally generated interference signals. Based on priorly known information of the transmitting signal and modeling of the coupling path, a replica interference signal can be subtracted at the input of the receiver, while the desired signal remains unaffected. However, the technique fails to deal with unknown interference signals. The adaption of modeling of the coupling path can also be power hungry at RF frequency.

Instead of the conventional LNA-first approach, recent works $[4,21]$ suggest directly connecting the receiver antenna to a CMOS passive mixer. This approach is referred to as mixer-first approach and is shown in Figure 5. There are four CMOS switch paths after the antenna, followed by RC low-pass filters (LPF). The switches are controlled by four path nonoverlapping $25 \%$ duty-cycle LO signals. The CMOS switches are favored for its high linearity, wide tuning range, and bidirectional response-translational property. The LPF at baseband is translated to a bandpass filter (BPF) at RF frequency and it can achieve much higher quality factor at RF than by using on-chip LC components. However, the interference filtering is limited by the switch resistance, accuracy of duty-cycle of LO signals, and the baseband filter order. It also suffers from limited isolation between RF and LO ports.

The simplified system architecture of a two-path feedforward cancellation receiver $[22,23]$ is shown in Figure 6(a). It is based on the upconversion of baseband filters. In the main path, both desired signal and unwanted interferer are amplified. In the auxiliary path after downconversion, the desired signal lies at DC and is filtered out by passing through a high-pass filter. The interferer is upconverted again and subtracted at the output of the LNA. The auxiliary path is identical to a high- $\mathrm{Q}$ notch filter centered at the desired signal frequency. This approach has a better input matching and better isolation between RF and LO port. However, the LNA linearity becomes a bottleneck for the tolerance of large interferers.

The simplified system architecture of a frequencytranslational noise-cancelling receiver [6] is shown in Figure 6(b). The receiver consists of two separate passive-mixerbased downconversion paths. The passive mixer downconverts the RF current to baseband. A transimpedance amplifier (TIA) then converts the in-band current back to voltage. Therefore the voltage gain is avoided at RF until baseband filtering is provided. The $3 \mathrm{~dB}$ noise figure brought by the matching resistor in the main path now can be cancelled by the auxiliary path. The Gm block is implemented as a CMOS inverter with small load impedance in order to handle large swings at the input. The frequency-translational noisecancelling receiver achieves to provide blocker tolerance, good OOB linearity, input matching, and low noise at the same time. However, the system's interference filtering is limited in the same way as in mixer-first approaches and also the IB linearity is poor in the presence of a large in-band blocker. 


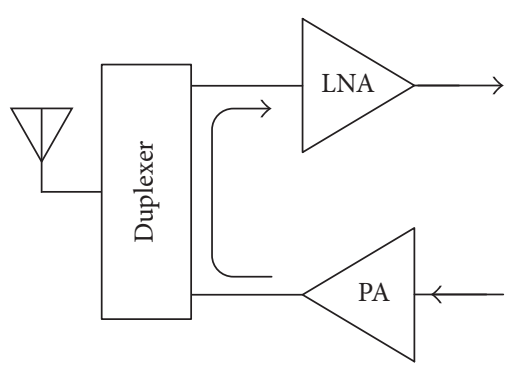

(a)

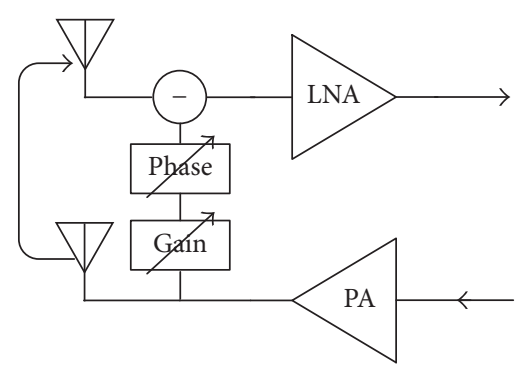

(b)

FIGURE 4: Simplified system architecture of (a) a FDD system with duplexer and (b) an analog cancellation method of local interferer.
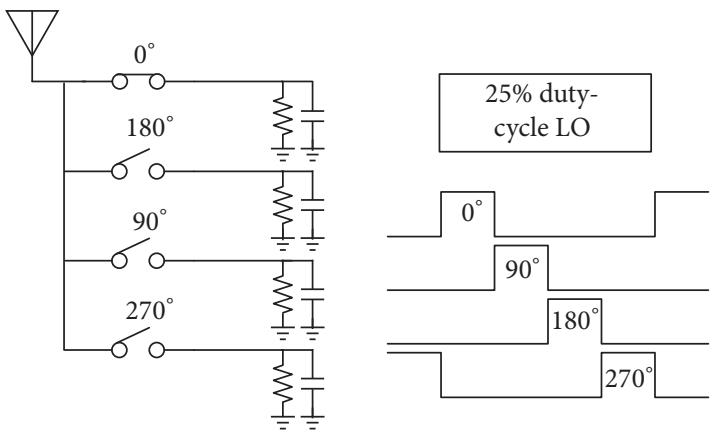

FIGURE 5: Model of 4-phase passive mixer with sampling capacitor, load resistor, and LO driving waveforms.

\section{Fundamental of Nonlinear Interference Suppression}

Nonlinear transfer functions [24-26] behave fundamentally different from linear transfer functions. The nonlinear transfer function does not obey the rule of superposition. The signals passing through a nonlinear system can undergo different operations. Also, nonlinear systems do not necessarily require large power consumption to handle a large signal. The fundamentals of a nonlinear system make it a possible candidate to deal with interference tolerance.

3.1. Time- and Frequency-Domain Behavior Analysis. The input and output signals in frequency and time domain for various conditions are illustrated in Figure 7. When a large signal (single-tone) is passing through an ideal linear system as shown in Figure 7(a), the signal is amplified linearly at the output. When the same input signal is passing through a conventional compressive nonlinear system as shown in Figure 7(b), the signal gets distorted at the output and 3rdorder harmonic is generated. Figure $7(\mathrm{c})$ shows a specially tailored nonlinear system with a third-order polynomial transfer function. When the large signal passes through, the fundamental tone of the large signal is completely removed at the output, while a $3 \mathrm{rd}$ harmonic is created. The process can be described using the following mathematical equations (2)-(6). The nonlinear transfer function is described as

$$
y(t)=c_{1} x(t)+c_{3} x(t)^{3} .
$$

The input signal is defined as a strong sinusoidal signal with amplitude $A_{\text {LS }}$ and frequency $\omega_{\text {LS }}$ :

$$
x(t)=A_{\mathrm{LS}} \sin \left(\omega_{\mathrm{LS}} t\right) .
$$

The output signal $y(t)$ is equal to

$$
\begin{aligned}
y(t)= & {\left[c_{1} A_{\mathrm{LS}}+c_{3} \frac{3 A_{\mathrm{LS}}^{3}}{4}\right] \sin \left(\omega_{\mathrm{LS}} t\right) } \\
& +c_{3} \frac{A_{\mathrm{LS}}^{3}}{4} \sin \left(3 \omega_{\mathrm{LS}} t\right) .
\end{aligned}
$$

If choosing

$$
c_{3}=-\frac{4 c_{1}}{3 A_{\mathrm{LS}}^{2}},
$$

the output becomes

$$
y(t)=-c_{1} \frac{A_{\mathrm{LS}}}{3} \sin \left(3 \omega_{\mathrm{LS}} t\right) .
$$

Furthermore, the nonlinear transfer function does not obey the rule of superposition. Therefore, the large signal accompanied with a (much weaker) signal passing through the nonlinear system can undergo different operations. The situation is illustrated in Figure 7(d). At the output, the large signal at fundamental frequency is suppressed completely, while the fundamental tone of the weak signal is amplified. 3 rd harmonics are generated for both large signal and weak signal. Besides, an intermodulation (IM) term arises with the same power as the output of the fundamental tone of the weak signal. The intermodulation term is the result of nonlinearity and convolution between input signals and introduces noise folding into the signal band [26], which will be discussed later.

Therefore, based on the envelope amplitude of the large interferer, the specially tailored nonlinear transfer function enables large interference suppression. When the amplitude of the large interferer changes, for example, modulated interferers, the nonlinear transfer function should be altered correspondingly to maintain the suppression. The nonlinear interference suppression can be considered as a notch filter in amplitude domain. The adaption of the nonlinear transfer function in amplitude domain is equivalent to the adaption of a frequency-domain notch filter. When the interferer 


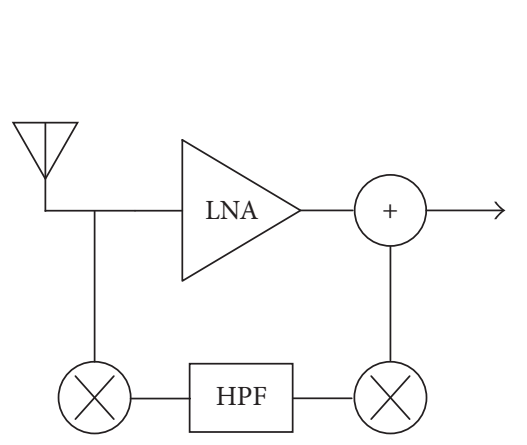

(a)

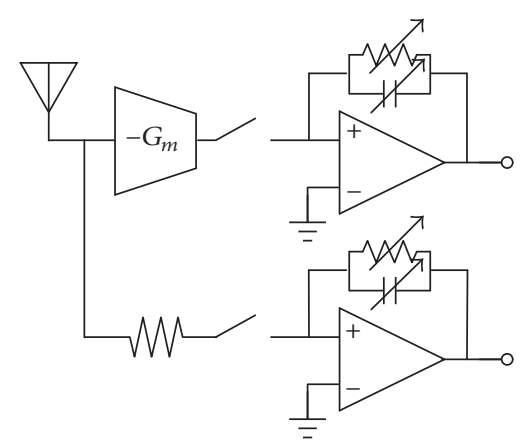

(b)

FIGURE 6: Simplified system architecture of (a) a two-path feedforward receiver and (b) a frequency-translational noise-cancelling receiver.
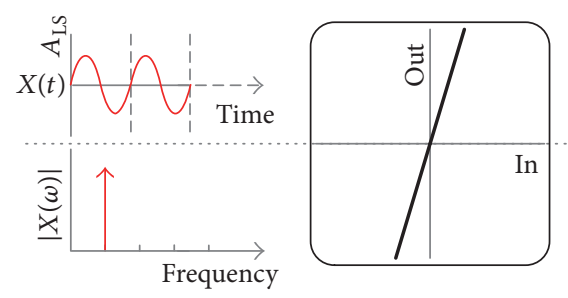

(a)
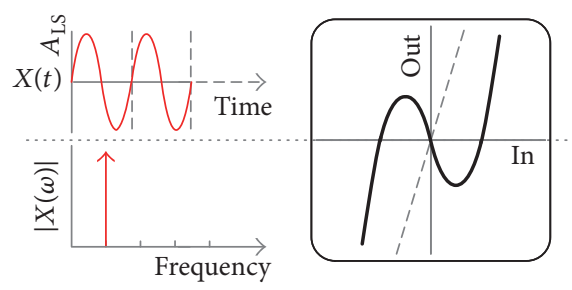

(c)
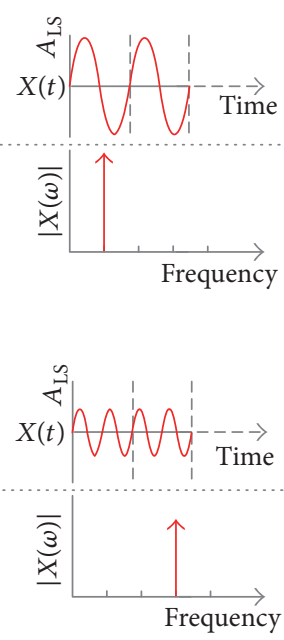

requency
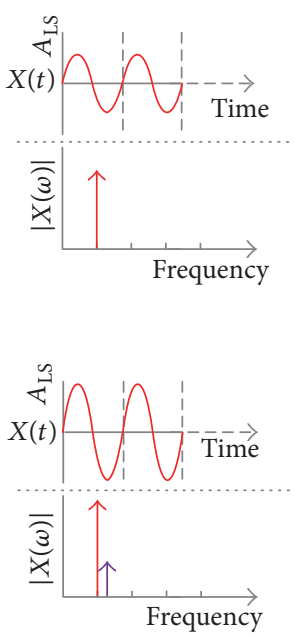

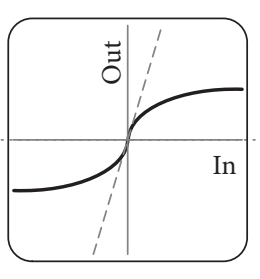

(b)

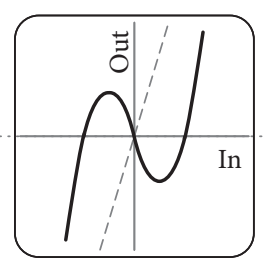

(d)
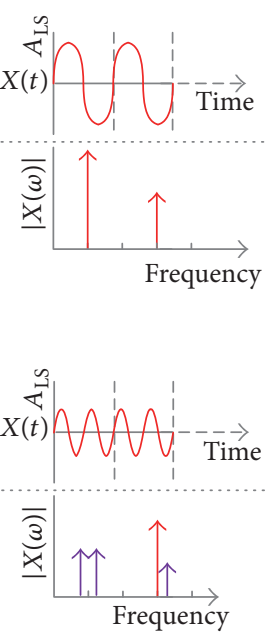

Frequency

Figure 7: Input and output of a large (single-tone) signal in frequency and time domain when passing through (a) ideal linear system, (b) conventional nonlinear system, (c) proposed nonlinear system, and (d) proposed nonlinear system accompanied with a weak signal.
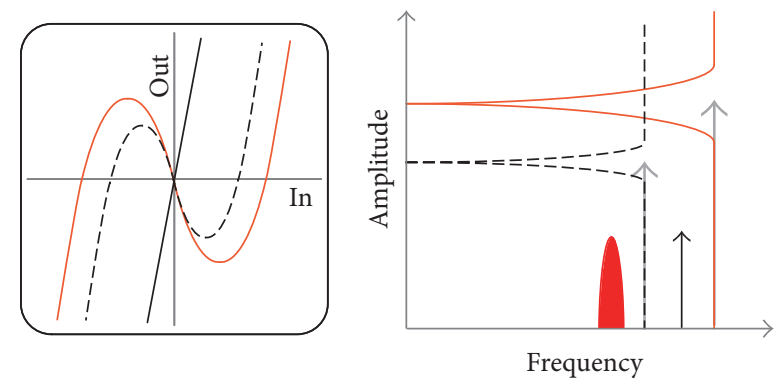

FIGURE 8: The adaption of nonlinear transfer function to maintain large signal suppression when the amplitude of large signal changes.

amplitude is similar to or smaller than the wanted signal, the transfer function can be switched to a linear one.

The adaption is shown in Figure 8. The nonlinear interference suppression relies on the amplitude discrimination between the strong signal and the weak signal. This is similar to frequency-domain filtering that relies on the relative frequency difference between input signals. In this way, the nonlinear interference suppression method enables frequency-independent filtering.

3.2. Nonlinear Transfer Characteristics for Large Interference Suppression. To derive the general requirements of a nonlinear transfer function for interference suppression, the input signal $x(t)$ is defined here:

$$
\begin{aligned}
x(t) & =\operatorname{Int}(t)+s(t), \\
\operatorname{Int}(t) & =A_{\mathrm{LS}}(t) \sin \left[\omega_{\mathrm{LS}} t+\varphi_{\mathrm{LS}}(t)\right], \\
s(t) & =A_{\mathrm{SS}}(t) \sin \left[\omega_{\mathrm{SS}} t+\varphi_{\mathrm{SS}}(t)\right], \\
A_{\mathrm{SS}} & =\left|A_{\mathrm{SS}}\right| \ll A_{\mathrm{LS}}=\left|A_{\mathrm{LS}}\right|,
\end{aligned}
$$

which consists of a strong interference signal $\operatorname{Int}(t)$ and a much weaker desired signal $s(t)$, with their corresponding amplitude, frequency, and phase information. The input is fed to a memoryless nonlinear system with transfer function $y=f(x)$. By applying Fourier analysis to the output signal, 
the effective gain of the fundamental component of the strong signal $G_{\mathrm{LS}}$ can be expressed as

$$
G_{\mathrm{LS}}=\frac{1}{A_{\mathrm{LS}} \cdot \pi} \int_{-\pi}^{\pi} f\left(A_{\mathrm{LS}} \sin \theta\right) \cdot \sin \theta d \theta .
$$

The transfer function $y=f(x)$ can be expressed as the sum of odd and even functions:

$$
f(x)=f_{\text {odd }}(x)+f_{\text {even }}(x) .
$$

For (8) to equal 0 , the transfer function can only consist of odd-order terms. Equation (8) becomes

$$
G_{\mathrm{LS}}=\frac{2}{A_{\mathrm{LS}} \cdot \pi} \int_{0}^{\pi} f_{\text {odd }}\left(A_{\mathrm{LS}} \sin \theta\right) \cdot \sin \theta d \theta .
$$

For the entire integration interval $\theta \in[0, \pi], \sin \theta \geq 0$ always exists, which means $f_{\text {odd }}\left(A_{\text {LS }} \sin \theta\right)$ must change sign in this interval. Therefore, the transfer function $y=f(x)$ must have at least one zero crossing in each interval $x \in\left[-A_{\mathrm{LS}}, 0\right)$ and $x \in\left(0, A_{\mathrm{LS}}\right]$. The third zero crossing is at the origin because of odd-order symmetry. Chebyshev polynomials are found to fulfill the requirement for such nonlinear transfer functions to provide large signal suppression [27]. It is also clear that the transfer function $f(x)$ is also a function of $A_{\mathrm{LS}}$, $y=f\left(x, A_{\mathrm{LS}}\right)$. If the input interference has varying envelope amplitude, for example, modulated interferer, the transfer function should be adapted according to the instantaneous value of the interferer envelope amplitude.

3.3. Consequence for Weak Desired Signal. The effective gain of the fundamental component of the weak signal $G_{S S}$ can be expressed as

$$
G_{S S}=\int_{-A_{\mathrm{LS}}}^{A_{\mathrm{LS}}} \frac{\partial f(x)}{\partial x} \cdot \operatorname{PDF}_{\text {sine }}(x) d x .
$$

From (11), $G_{S S}$ is a function of $A_{\mathrm{LS}}$, probability density function (PDF) of the sinusoidal $\operatorname{Int}(t)$ and $f(x)$. Both $G_{\mathrm{LS}}$ and $G_{S S}$ depend on the amplitude of $\operatorname{Int}(t)$ and transfer function $y=f(x)$, while the phase or frequency of $\operatorname{Int}(t)$ and $s(t)$ is irrelevant. In case there is no large interference or only small interference exists, the system transfer function can be switched to a linear one, resulting in conventional compressive receiver behavior.

Given the expression of effective gain of strong signal and weak signal, the output of the nonlinear transfer function using method described in [28] becomes

$$
\begin{aligned}
y(t)= & G_{\mathrm{LS}} \cdot \operatorname{Int}(t)+\frac{1}{2}\left[A_{\mathrm{LS}} \cdot \frac{\partial G_{\mathrm{LS}}}{\partial A_{\mathrm{LS}}}+G_{\mathrm{LS}}\right] \cdot s(t) \\
& +\frac{1}{2}\left[A_{\mathrm{LS}} \cdot \frac{\partial G_{\mathrm{LS}}}{\partial A_{\mathrm{LS}}}-G_{\mathrm{LS}}\right] \cdot \operatorname{IM}(t),
\end{aligned}
$$

in which the first term is the remaining residue of strong interference, the second term is the desired signal output, and the third term is the intermodulation (IM) product between the strong and weak signal. The IM product is given by

$\operatorname{IM}(t)$

$$
=A_{\mathrm{SS}}(t) \sin \left[\left(2 \omega_{\mathrm{LS}}-\omega_{\mathrm{SS}}\right) t+2 \varphi_{\mathrm{LS}}(t)-\varphi_{\mathrm{SS}}(t)\right] .
$$

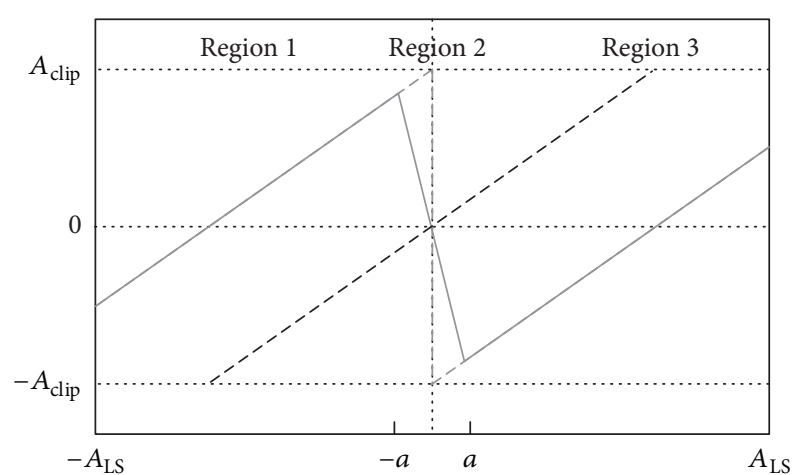

FIGURE 9: Zig-zag transfer function (grey) for an interferer with envelope amplitude of $A_{\mathrm{LS}}$. By limiting $a$ to zero, the zig-zag function has an infinite slope in the zero-transition region 2. The creation of the zig-zag transfer function can be considered as shifting the linear transfer (black) in $y$-axis in opposite directions in regions 1 and 3.

With complete interference suppression, $G_{\mathrm{LS}}=0$, the desired signal output and the IM term have the same magnitude, which explains the frequency-domain behavior in Figure 7(d). When the nonlinear transfer function is set for full suppression at amplitude $A_{\mathrm{LS}}$, the weak signal at $\omega_{\mathrm{SS}}$ is mirrored to the frequency component at $2 \omega_{\mathrm{LS}}-\omega_{\mathrm{SS}}$. On the other hand, the noise at $2 \omega_{\mathrm{LS}}-\omega_{\mathrm{SS}}$ is folded to the desired signal frequency. If the frequency difference between strong signal and small signal $\left|\omega_{\mathrm{LS}}-\omega_{\mathrm{SS}}\right|$ is small, the cross talk introduces noise penalty of $3 \mathrm{~dB}$. If the frequency difference is large and the circuit is narrowband, the noise penalty is less than $3 \mathrm{~dB}$.

3.4. Transfer-Specific Characteristics. To further analyze the nonlinear operation and consequences, a specific nonlinear transfer function is chosen here. As shown in Figure 9, the zig-zag function is created by high order Chebyshev polynomials. By limiting $a$ to 0 , it has infinite slope in the zero-transition region 2 . The mathematic expression of the zig-zag function is

$$
f(x)= \begin{cases}G_{\text {lin }} \cdot x+A_{\text {clip }}, & \text { if } x<-a \\ \left(G_{\text {lin }}-\frac{A_{\text {clip }}}{a}\right) \cdot x, & \text { if }-a \leq x \leq a \\ G_{\text {lin }} \cdot x-A_{\text {clip }}, & \text { if } x>a,\end{cases}
$$

where $G_{\text {lin }}$ is the slope of the function in regions 1 and 3. For complete suppression at amplitude $A_{\mathrm{LS}}$, based on (8),

$$
G_{\mathrm{LS}}=G_{\text {lin }}-\frac{4 A_{\text {clip }}}{\pi A_{\mathrm{LS}}}
$$

$$
A_{\text {clip,supp }}=\frac{\pi}{4} A_{\mathrm{LS}} G_{\text {lin }} .
$$


Based on (11), assuming a unity amplitude interferer for simplicity, the first-order small signal gain $G_{S S, 1}$ becomes

$$
\begin{aligned}
G_{\mathrm{SS}, 1}= & \int_{-1}^{-a} G_{\text {lin }} \cdot \frac{d x}{\pi \sqrt{1-x^{2}}} \\
& +\int_{-a}^{a}\left(G_{\text {lin }}-\frac{A_{\text {clip }}}{a}\right) \cdot \frac{d x}{\pi \sqrt{1-x^{2}}} \\
& +\int_{a}^{1} G_{\operatorname{lin}} \cdot \frac{d x}{\pi \sqrt{1-x^{2}}} \\
= & \frac{G_{\text {lin }}}{\pi}\left[\pi-\frac{2 \pi}{4 a} \arcsin (a)\right], \\
\lim _{a \rightarrow 0} G_{\mathrm{SS}, 1}= & \frac{G_{\text {lin }}}{2} .
\end{aligned}
$$

The dependence of $G_{S S, 1}$ on the value $a$ of the zero-transition region 2 in the zig-zag function is shown in Figure 10. The normalized small signal gain varies little with change of value $a$, which can be explained by the weighting function of the PDF of the sinusoidal input waveform in (11). Because the PDF of a sinusoidal waveform is high near the edges and low in the center, the circuit is forced to operate mostly near the edges in large signal operation. So the derivative of the zig-zag function in regions 1 and 3 plays a bigger role in $G_{S S, 1}$. Therefore, the weak desired signal will experience amplification if $G_{\text {lin }}>2$ and (15) is satisfied. However the large signal suppression is decreasing with increasing $a$, since the zig-zag function deviates more from the original setting.

The third-order small signal gain $G_{\mathrm{SS}, 3}$, assuming $a$ is approaching zero, can be calculated using

$$
\lim _{a \rightarrow 0} G_{S S, 3}=\int_{-A_{\mathrm{LS}}}^{A_{\mathrm{LS}}} \frac{\partial^{3} f(x)}{\partial^{3} x} \cdot \frac{d x}{\pi \sqrt{1-x^{2}}}=-\frac{G_{\mathrm{lin}}}{16 A_{\mathrm{LS}}^{2}},
$$

leading to:

$$
V_{\mathrm{IIP} 3}=\sqrt{\left|\frac{4 G_{\mathrm{SS}, 1}}{3 G_{\mathrm{SS}, 3}}\right|}=\sqrt{\frac{32}{3}} \cdot A_{\mathrm{LS}} .
$$

So, the $\mathrm{IIP}_{3}$ of the nonlinear system using the ideal zigzag transfer function is approximately $10 \mathrm{~dB}$ higher than the amplitude of strong interferer signal.

3.5. Noise Properties of Noiseless Nonlinear Transfers. Based on (11), the output signal power can be calculated as

$$
\begin{aligned}
& \text { Output signal power }=\left|A_{\mathrm{SS}} \cdot G_{\mathrm{SS}}\right|^{2} \\
& =\left|A_{\mathrm{SS}}\right|^{2} \cdot\left|\int_{-A_{\mathrm{LS}}}^{A_{\mathrm{LS}}} \frac{\partial f(x)}{\partial x} \cdot \frac{d x}{\pi \sqrt{A_{\mathrm{LS}}^{2}-x^{2}}}\right|^{2} .
\end{aligned}
$$

As shown in Figure 9, the derivative of the zig-zag transfer function changes sign between different regions, resulting from the requirement for three zero crossings. The circuit will experience constructive behavior in regions 1 and 3 and destructive behavior in region 2 .

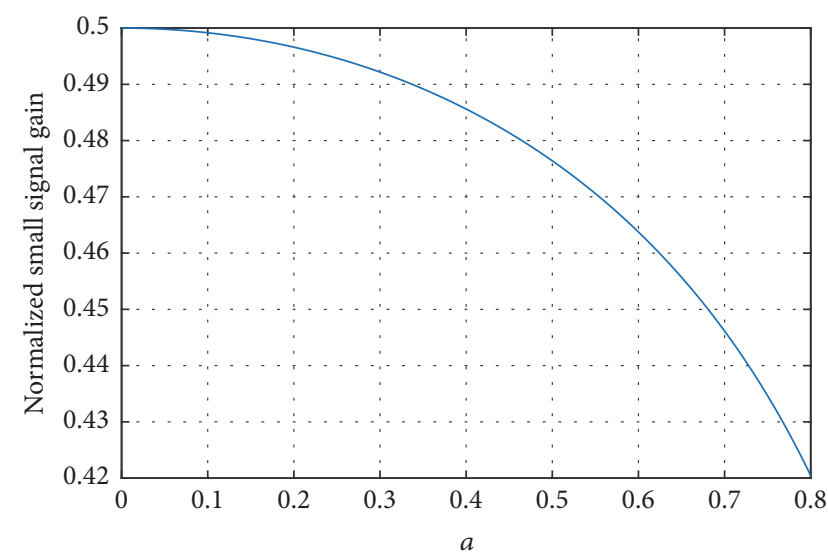

FIGURE 10: The influence of zero-transition region $a$ on normalized small signal gain $G_{\mathrm{SS}, 1}$ using zig-zag transfer function for nonlinear interference suppression.

However, for output noise power, all regions lead to additive behavior because the noise is white. So the output noise power can be calculated by

$$
\begin{aligned}
& \text { Output noise power } \\
& \qquad \int_{-A_{\mathrm{LS}}}^{A_{\mathrm{LS}}}\left|\frac{\partial f(x)}{\partial x} \cdot v_{n}\right|^{2} \cdot \frac{d x}{\pi \sqrt{A_{\mathrm{LS}}^{2}-x^{2}}},
\end{aligned}
$$

where $v_{n}$ is the standard deviation of the Gaussian noise voltage.

Based on (19) and (20), the noise folding penalty brought by the nonlinear transfer function can be calculated by (21), in which the numerator is the output noise power and the denominator is the output signal power when the input signal is equivalent to noise voltage:

$$
\begin{aligned}
F & =\frac{\text { Output noise power }}{\text { Output signal power }\left.\right|_{A_{\mathrm{ss}}=v_{n}}} \\
& =\frac{\left|v_{n}\right|^{2} \cdot \int_{-A_{\mathrm{LS}}}^{A_{\mathrm{LS}}}|\partial f(x) / \partial x|^{2} \cdot\left(d x / \pi \sqrt{A_{\mathrm{LS}}^{2}-x^{2}}\right)}{\left|A_{\mathrm{SS}}\right|^{2} \cdot\left|\int_{-A_{\mathrm{LS}}}^{A_{\mathrm{LS}}}(\partial f(x) / \partial x) \cdot\left(d x / \pi \sqrt{A_{\mathrm{LS}}^{2}-x^{2}}\right)\right|^{2}} .
\end{aligned}
$$

If the transfer function $y=f(x)$ is a noiseless linear function, that is, $f(x)=c_{1} x$, as shown in Figure 9 (black), (21) becomes

$$
F_{\text {linear }}=\frac{\int_{-A_{\mathrm{LS}}}^{A_{\mathrm{LS}}}\left|c_{1}\right|^{2} \cdot\left(d x / \pi \sqrt{A_{\mathrm{LS}}^{2}-x^{2}}\right)}{\left|\int_{-A_{\mathrm{LS}}}^{A_{\mathrm{LS}}} c_{1} \cdot\left(d x / \pi \sqrt{A_{\mathrm{LS}}^{2}-x^{2}}\right)\right|^{2}}=1,
$$

since there is no spectrum mirroring and high frequency terms in a linear transfer function. If the transfer function is a 
noiseless 3rd Chebyshev polynomial as shown in (2) and (5), (21) becomes

$$
\begin{aligned}
F_{3 \mathrm{rd}} & =\frac{\int_{-A_{\mathrm{LS}}}^{A_{\mathrm{LS}}}\left|c_{1}-4 c_{1} x^{2}\right|^{2} \cdot\left(d x / \pi \sqrt{A_{\mathrm{LS}}^{2}-x^{2}}\right)}{\left|\int_{-A_{\mathrm{LS}}}^{A_{\mathrm{LS}}}\left(c_{1}-4 c_{1} x^{2}\right) \cdot\left(d x / \pi \sqrt{A_{\mathrm{LS}}^{2}-x^{2}}\right)\right|^{2}} \\
& =3 .
\end{aligned}
$$

That is equal to a noise figure $(\mathrm{NF})$ of $4.77 \mathrm{~dB}$, resulting from the noise folding from the mirroring product and 3rdharmonic component.

If the transfer function is a noiseless zig-zag nonlinear function as shown in (14) and (15) and shown in Figure 9 (grey), assuming a unity amplitude interferer for simplicity, the numerator in (21) becomes

$$
\begin{aligned}
\text { Numerator }= & \int_{-1}^{-a}\left|G_{\operatorname{lin}}\right|^{2} \cdot \frac{d x}{\pi \sqrt{1-x^{2}}} \\
& +\int_{-a}^{a}\left|G_{\operatorname{lin}}-\frac{\pi \cdot G_{\operatorname{lin}}}{4 a}\right|^{2} \cdot \frac{d x}{\pi \sqrt{1-x^{2}}} \\
& +\int_{a}^{1}\left|G_{\operatorname{lin}}\right|^{2} \cdot \frac{d x}{\pi \sqrt{1-x^{2}}} \\
= & \left|G_{\operatorname{lin}}\right|^{2}\left[1+\left(\frac{\pi}{8 a^{2}}-\frac{1}{a}\right) \cdot \arcsin (a)\right] .
\end{aligned}
$$

The denominator in (21) becomes

$$
\begin{aligned}
& \text { Denominator }=\mid \int_{-1}^{-a} G_{\operatorname{lin}} \cdot \frac{d x}{\pi \sqrt{1-x^{2}}} \\
& +\int_{-a}^{a}\left(G_{\operatorname{lin}}-\frac{\pi \cdot G_{\operatorname{lin}}}{4 a}\right) \cdot \frac{d x}{\pi \sqrt{1-x^{2}}} \\
& \quad+\left.\int_{a}^{1} G_{\operatorname{lin}} \cdot \frac{d x}{\pi \sqrt{1-x^{2}}}\right|^{2}=\left|G_{\operatorname{lin}}\right|^{2} \cdot[1 \\
& \left.\quad-\frac{\arcsin (a)}{2 a}\right]^{2} .
\end{aligned}
$$

The noise factor of a noiseless zig-zag transfer function becomes

$$
F_{\text {zig-zag }}=\frac{1+\left(\pi / 8 a^{2}-1 / a\right) \cdot \arcsin (a)}{1-(\arcsin (a)) / 2 a} .
$$

Equation (26) is plotted in $\mathrm{dB}$ scale in Figure 11. By decreasing $a$, the NF increases steadily. This is because, by decreasing $a$, the nonlinear zig-zag function has sharper zero transition in region 2, introducing more noise from high frequency components folding onto the signal frequency. By increasing $a$, NF keeps decreasing as fewer high frequency noise components fold to the signal band. The NF almost decreases to zero at large $a$ value. However, this result is meaningless since the transfer function at larger $a$ value is totally different from an ideal zig-zag function and (15) is no longer satisfied.

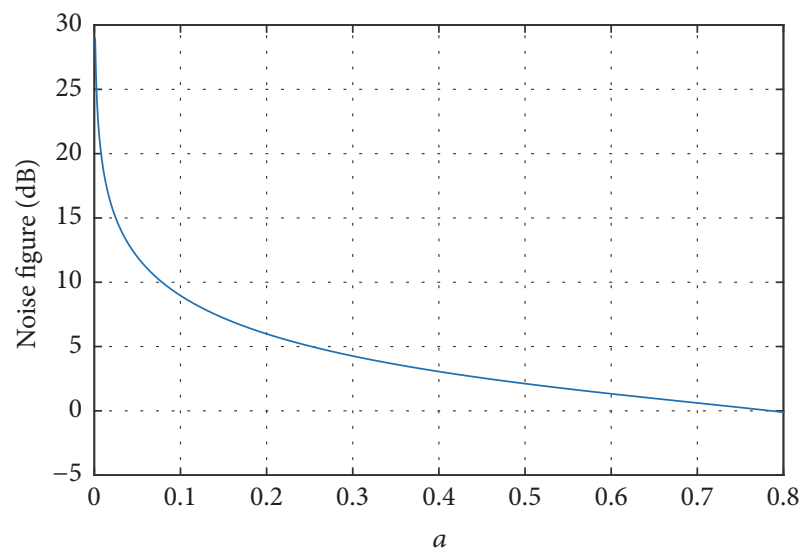

FIGURE 11: The influence of zero-transition region $a$ on noise figure (NF) using zig-zag transfer function for nonlinear interference suppression.

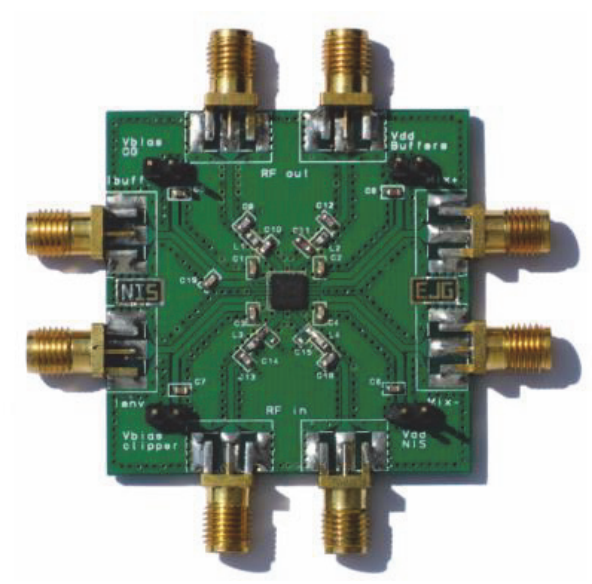

FIGURE 12: PCB including the nonlinear interference suppression (NIS) IC implementation.

\section{Nonlinear System Modeling and Analysis}

4.1. System Architecture. A $1.8 \mathrm{GHz} \mathrm{RF}$ amplifier with linear mode and nonlinear mode operation was implemented in a $140 \mathrm{~nm}$ CMOS technology. The nonlinear mode operation is enabled for frequency-independent interference suppression, while the linear mode is for linear amplification when no large interference is present. In the presence of a 0 to $11 \mathrm{dBm}$ interferer, the interferer is suppressed by more than $39 \mathrm{~dB}$ [27]. The PCB including the nonlinear RF amplifier IC implementation is shown in Figure 12.

Figure 13(a) shows the system diagram of applying the nonlinear method to the multiradio coexistence in the same device. The highlighted are the key subblocks in the nonlinear receiver system, including NIS, cross-correlation mixer, LPF, ADC, DAC, Magnitude, and NIS Control subblocks. To derive the exact amplitude information of the interferer, the baseband $I$ and $Q$ signal from the transmitter are fed to the Magnitude subblock. The Magnitude subblock models the path loss between transmitter baseband and receiver antenna and is connected to a subblock named NIS Control. 


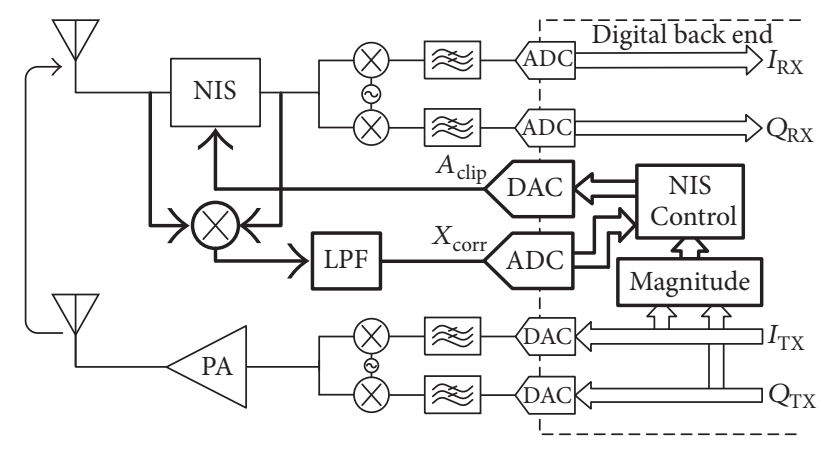

(a)

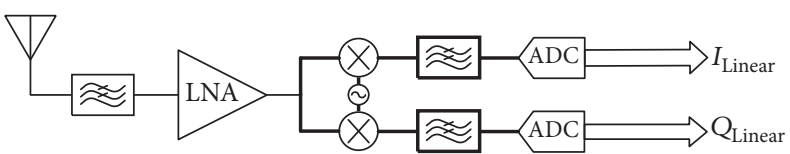

(b)

FIGURE 13: The system diagram of (a) the proposed NIS operation in a multiradio platform and (b) a conventional narrowband receiver with off-chip SAW filter.

The NIS Control subblock interfaces a DAC that steers the control signal $A_{\text {clip }}$ to the NIS subblock, where the nonlinear suppression is performed. This path can be recognized as a feedforward path. Based on the interferer information from transmitter baseband, the feedforward path gives the correct control signal with accuracy and speed.

On the other hand, a feedback path is also needed to model the coupling changes between the transmitter antenna and the receiver antenna. Therefore, a mixer is placed around the NIS subblock to provide cross-correlation between the input and output of the NIS subblock. Assuming the interferer is the dominant signal, the cross-correlation measures how much the residue interference remains after nonlinear suppression, representing the errors in control signal $A_{\text {clip. }}$. The cross-correlation signal is fed back to the NIS Control subblock to form a feedback path. The feedback path only requires low speed because environment changes slowly, while the feedforward path should be fast enough to update the control signal with the changes of the interferer envelope amplitude.

A conventional narrowband receiver with off-chip SAW filter is shown in Figure 13(b) for a comparison of interference tolerance with the nonlinear receiver system in a later section. Both receivers are assumed with direct conversion architecture.

4.2. NIS Modeling and Analysis. The nonlinear receiver system is modeled in Advanced Design System (ADS). The NIS, cross-correlation mixer, NIS Control, and Magnitude subblocks are modeled with symbolically defined devices. The downconversion mixer uses ideal mixer component with ideal I/Q demodulation. The baseband filter is set as a 4 thorder Butterworth filter with a bandwidth determined by the data rate of the wanted signal. The amplifier is set as ideal $30 \mathrm{~dB}$ gain broadband amplifier.

16-QAM modulation scheme is used for both the interferer and the desired signal with raised cosine pulse shaping and a roll-off factor of 0.5. The basebands $I$ and $Q$ of both signals have a data rate of $20 \mathrm{Mbps}$. The weak signal lies at $1.825 \mathrm{GHz}\left(f_{s}\right)$ with $-50 \mathrm{dBm}$ power and the strong signal at $1.870 \mathrm{GHz}\left(f_{i}\right)$ with $10 \mathrm{dBm}$ power. The frequency separation $\Delta f$ between the input signals is $15 \mathrm{MHz}$. The input spectrum is shown in Figure 14(a).
TABLE 1: Interference suppression limitations.

\begin{tabular}{lccc}
\hline $\begin{array}{l}\text { Interference } \\
\text { power }(\mathrm{dBm})\end{array}$ & $\begin{array}{c}\text { Interference } \\
\text { suppression at RF } \\
(\mathrm{dB})\end{array}$ & EVM $(\%)$ & SNR $(\mathrm{dB})$ \\
\hline-30 & 20 & 14.4 & 17 \\
-20 & 42 & 3.7 & 29 \\
-10 & 56 & 1.9 & 34 \\
0 & 66 & 1.9 & 34 \\
10 & 80 & 2.9 & 31 \\
\hline
\end{tabular}

The output spectrum of the NIS subblock is shown in Figure 14(b). As shown in Figure 14(b), the fundamental tone of the weak signal remains, while the fundamental tone of the interferer is totally suppressed. The intermodulation terms lies at $2 f_{i}-f_{s}$ and its bandwidth depends on the convolution of the wanted signal and the interferer. The interference suppression by NIS subblock is about $80 \mathrm{~dB}$. After passing to the baseband, the signal is amplified by 1000 times, while the interferer residue and intermodulation term are further suppressed, as shown in Figure 14(c). The constellation diagram of the baseband output is shown in Figure 15. The corresponding error vector magnitude (EVM) is $2.82 \%$, which corresponds to a signal-to-noise ratio (SNR) of $31 \mathrm{~dB}$. 16-QAM modulation requires a SNR of $17.6 \mathrm{~dB}$ to achieve a symbol-to-error ratio (SER) of $10^{-3}$ [29], which is achieved here.

As pointed out before, the interference suppression at RF stage by nonlinear transfer function is based on the amplitude discrimination between the interferer and the wanted signal. To illustrate the influence of the relative power ratio, the input signals are kept the same except the interferer power is swept from $-30 \mathrm{dBm}$ to $10 \mathrm{dBm}$. The results of RF suppression, EVM, and SNR at baseband output are shown in Table 1. For an increasing interference power, larger interference suppression at RF is achieved with a better EVM and SNR at baseband output.

The probability densify function (PDF) of the instantaneous power of the modulated interferers for input power and the PDF of the wanted signal are shown in Figure 16. It is observed that when the relative power ratio between the 


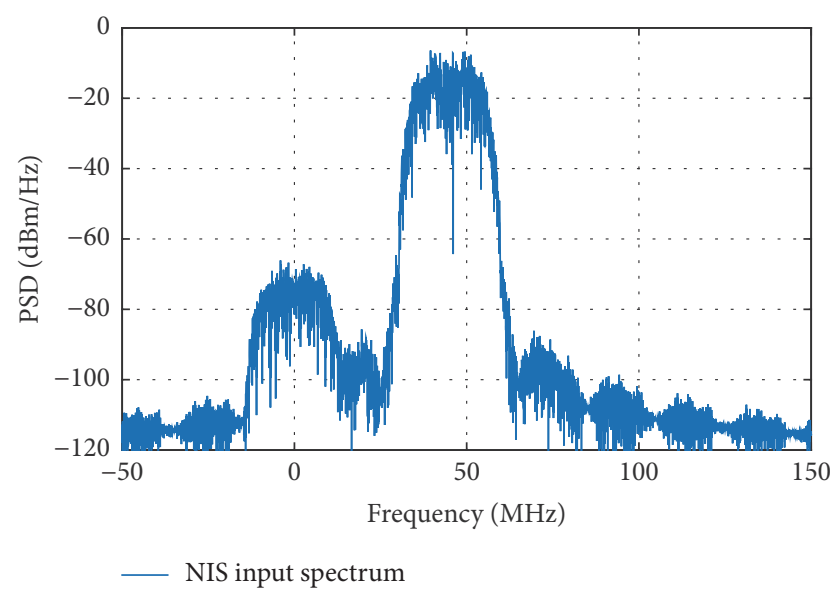

(a)

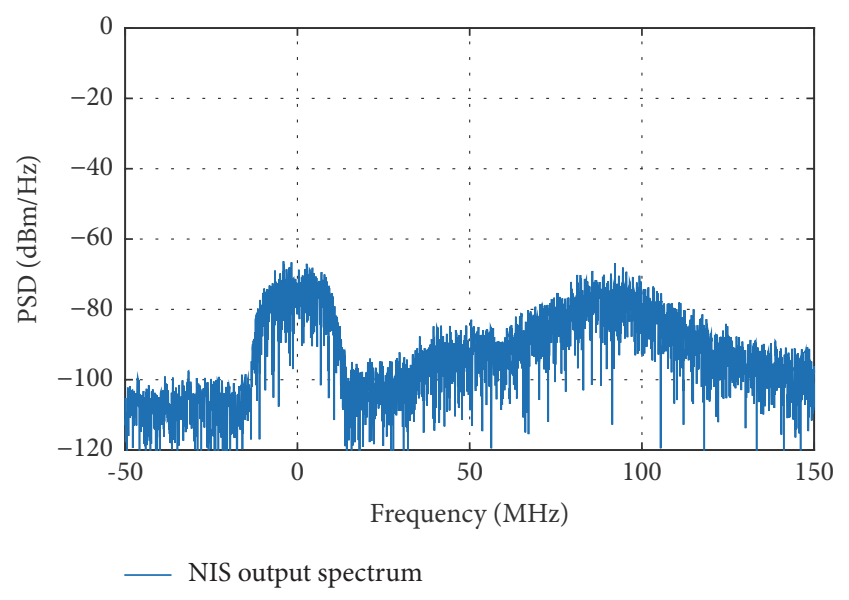

(b)

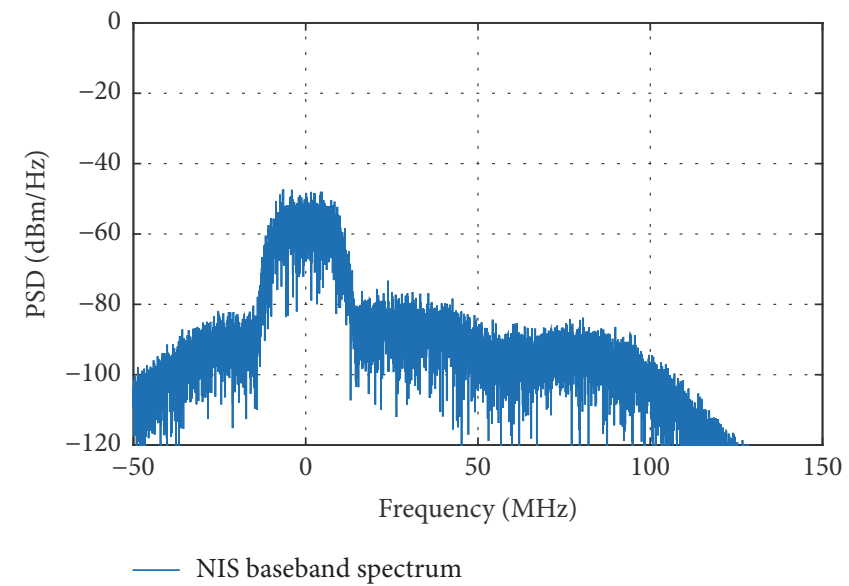

(c)

FIGURE 14: The frequency spectrum of (a) input at receiving antenna, (b) output of NIS subblock, and (c) baseband output.

interferer and the wanted signal is as low as $20 \mathrm{~dB}$, the PDF of the instantaneous power of the interferer overlaps with that of the wanted signal. Therefore the nonlinear system is incapable of distinguishing one from another, thus leading to the limited suppression performance of the interferer and distortion of the desired signal. When the relative power is even lower, the NIS subblock output is completely flooded by noise and distortion.

The limitation for complete interference suppression also comes from baseband filtering for signals outside the baseband bandwidth. The baseband filtering is determined by the baseband filter design such as order and power.

The cross-correlation mixer output spectrum is shown in Figure 17. The output spectrum has convolution products at DC and intermodulation frequency. As the interferer is totally suppressed in this case, the output spectrum at DC and intermodulation frequency is low. If the interferer is only partially suppressed, the mixer output at DC will reflect the error in the control signal. The intermodulation term would also be bigger but discarded because the speed of the feedback path is slow.

For simplicity, the 3rd-order harmonic generated by the nonlinear receiver with nonlinear transfer function is not shown here. It can be removed by frequency-domain filters and harmonic rejection mixers to avoid harmonic mixing.

4.3. Comparison of Linear and Nonlinear Receiver. The system diagrams of a nonlinear receiver system with proposed nonlinear interference suppression and a conventional narrowband linear receiver are shown previously in Figure 13. The downconversion mixer and baseband circuitry are set as exactly the same for a fair comparison on interference tolerance. The low noise amplifier (LNA) in the conventional linear narrowband receiver is configured as ideal component and unit gain. That is same for the setting of nonlinear transfer function to provide unit gain for the desired signal. Therefore, in the modeling of the conventional linear narrowband receiver in ADS, the LNA is simply removed. The SAW filter after the antenna is set with a center frequency at the signal frequency, a passband bandwidth of $200 \mathrm{MHz}$ and a stopband bandwidth of $220 \mathrm{MHz}$ with $40 \mathrm{~dB}$ attenuation. Again the same input signals are used here, a 16-QAM interferer with $10 \mathrm{dBm}$ power and a 16-QAM weak wanted signal with $-50 \mathrm{dBm}$ power.

The signal-to-interference ratio (SIR) is used here to characterize the interference tolerance and influences on 


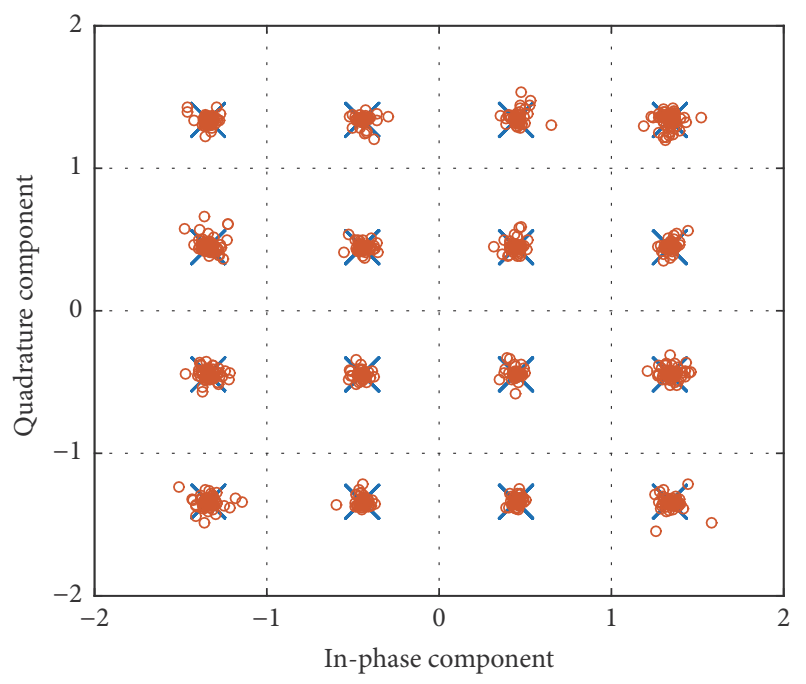

FIGURE 15: Baseband output constellation diagram of the nonlinear receiver system.

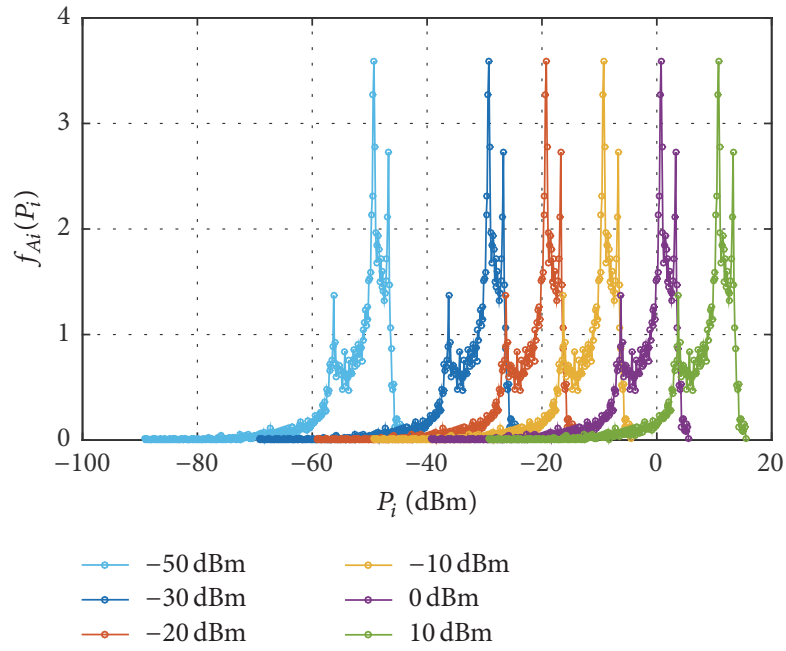

FIGURE 16: Probability density function of the instantaneous power of the interferer from $-30 \mathrm{dBm}$ to $10 \mathrm{dBm}$ and probability density function of the instantaneous power of the wanted signal with $-50 \mathrm{dBm}$ power (light blue).

the linear and nonlinear receiver. Initially the SIR at the input of the receiver is $-60 \mathrm{~dB}$. The SIR at the input of baseband ADCs should be at least higher than zero, so that the signal is amplified while the interferer is largely suppressed. The suppression of interference signal before $\mathrm{ADC}$ is beneficial since it alleviates signal aliasing. Besides, the residue interference also needs extra $\mathrm{ADC}$ resolution bits to quantize the total input at baseband A DCs. According to [14], one additional ADC bit should be added for every $6 \mathrm{~dB}$ decrement of SIR, which will lead to a huge increase in ADC power consumption.

The comparison of SIR at baseband output of the linear receiver and the nonlinear receiver versus frequency separation between large interference and wanted signal is shown in Figure 18. The SIR of the linear receiver is achieved completely

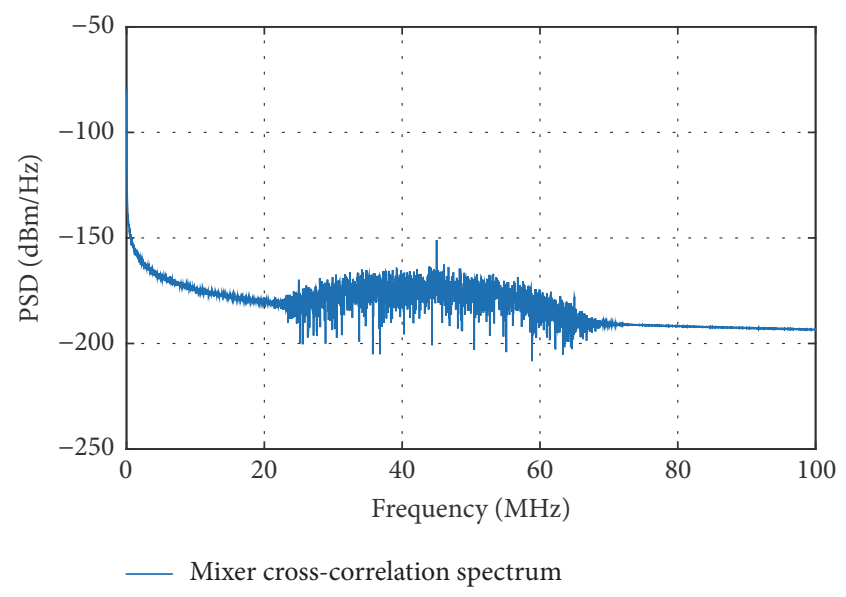

FIGURE 17: The frequency spectrum of cross-correlation mixer output.

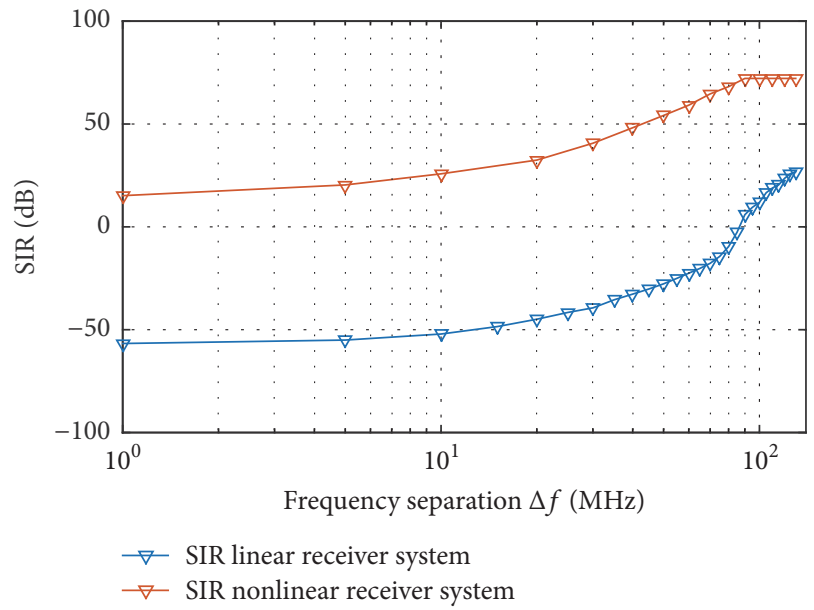

FIGURE 18: SIR of baseband output of the linear receiver (blue) and the nonlinear receiver (red) versus the frequency separation between input signals.

by the SAW filter and baseband 4th order LPF. Between the edge of the passband and stopband of the SAW filter, the SIR of the linear receiver increase sharply as the interference falls outside the SAW filter passband. The SIR of the nonlinear receiver is achieved with the help of nonlinear interference suppression. The SIR of the nonlinear receiver has a positive value and is higher than the SIR of the linear receiver. Since the nonlinear interference suppression is frequencyindependent, the increase of SIR curve of the nonlinear receiver is similar to that of the linear receiver. However, since both input signals have $20 \mathrm{MHz}$ bandwidth, both SIR do not increase exactly like a 4 th-transfer function. As the frequency separation $\Delta f$ between the input signals increases, the available suppression provided by the baseband filter is limited by the noise floor, which leads to a saturated value of SIR for the nonlinear receiver. The SNR at the baseband output of the nonlinear receiver system is shown in Figure 19. The SNR is $20.2 \mathrm{~dB}$ at $\Delta f$ of $1 \mathrm{MHz}$ and converges to $42 \mathrm{~dB}$ when $\Delta f$ increases. 


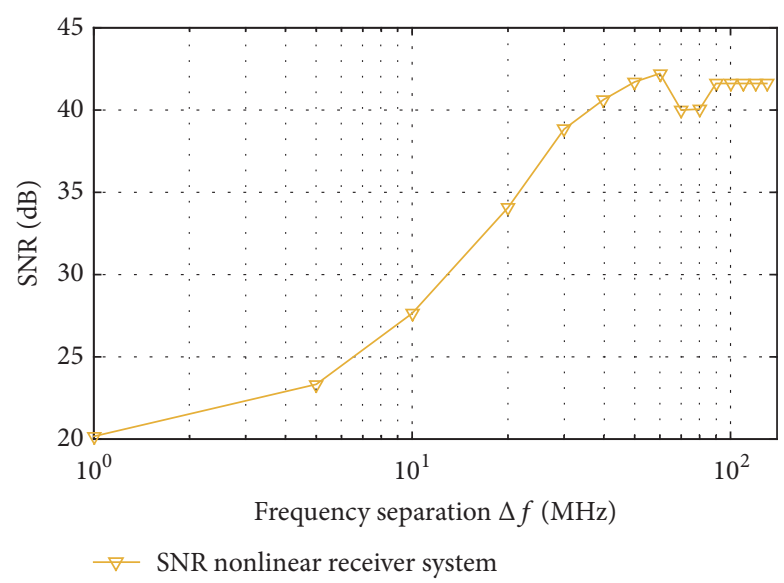

FIGURE 19: SNR of baseband output of the nonlinear receiver versus the frequency separation.

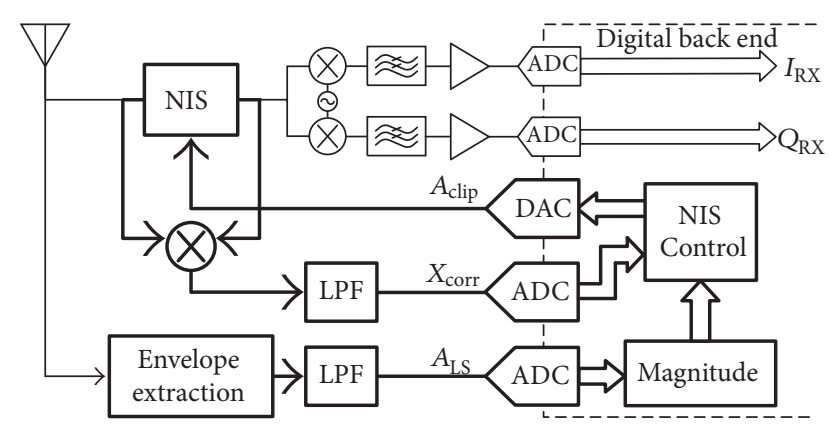

FIGURE 20: The system diagram of the proposed NIS operation for general interference suppression.

\section{NIS Modeling for General Interference}

5.1. System Architecture for General Interference Suppression. To extend the nonlinear method to the suppression of general large interference, the envelope amplitude of the interference needs to be extracted. The system architecture for general large interference suppression is shown in Figure 20.

The feedforward path starts from the receiving antenna and consists of an envelope extraction subblock followed by LPF to derive the amplitude information. The envelope extraction subblock can be implemented as self-mixing mixers or diodes. The extracted envelope contains noise received by antenna, the envelope information of the desired signal, and the envelope information of interferer. However, as the focus of this work is the coexistence of large interferer and weak desired signal, the envelope of the wanted signal behaves as noise and small disturbance to the control signal $A_{\text {clip }}$.

5.2. NIS Modeling and Analysis. Here the desired signal is assumed as a 16-QAM modulated signal with raised cosine pulse shaping and a roll-off factor of 0.5 . The baseband $I$ and $Q$ signals have a data rate of $10 \mathrm{Mbps}$. The interference is a QPSK signal with same pulse shaping and a data rate of $2.5 \mathrm{Mbps}$ for basebands $I$ and $Q$. The weak signal lies at $1.825 \mathrm{GHz}\left(f_{s}\right)$ with $-50 \mathrm{dBm}$ power and the strong signal at
1. $870 \mathrm{GHz}\left(f_{i}\right)$ with $10 \mathrm{dBm}$ power. The input spectrum of the nonlinear receiver is shown in Figure 21(a).

The output spectrum of NIS subblock is shown in Figure 21(b). It is observed that the fundamental component of the weak signal remains, while the interference signal is only partially suppressed. The nonlinear interference suppression at RF is about $40 \mathrm{~dB}$. Therefore more filtering at baseband is needed. The baseband filter order is increased to 6 th order. After passing the baseband circuitry, the signal is amplified by $30 \mathrm{~dB}$, while the interference residue and the intermodulation term are further suppressed, as shown in Figure 21(c). The constellation diagram of the baseband output is shown in Figure 22. The EVM at the baseband output is 5.4\%, which corresponds to a SNR of $25 \mathrm{~dB}$.

The limited suppression at RF is a result of inaccuracy of the extracted interference envelope. Figure 23 shows the comparison of frequency spectrum between the input interferer envelope and the control signal for NIS subblock. The spectrum of the control signal is quite the same at DC compared to the interferer envelope spectrum. However there is an intermodulation term at $\Delta f$. The intermodulation term is the result of convolution between the input signals in frequency domain. Thus a LPF is needed after the envelope extraction subblock to filter out the intermodulation term. However there is a tradeoff between the filtering of the intermodulation term and the delay introduced by the filter. The filter bandwidth could be set small to filter out the intermodulation term completely, while it will introduce a big delay on the control signal. On the other hand, if the filter bandwidth is set large so that there is little delay introduced, the residue of the intermodulation term brings error to the control signal.

The amount of interference suppression versus LPF bandwidth $\left(F_{\mathrm{LPF}}\right)$ is summarized in Table 2. With larger $F_{\mathrm{LPF}}$, smaller delay is introduced, and the interference suppression is increasing. To compensate the delay of the control signal, a delay block can be added before the input of NIS subblock. With the corresponding delay compensated, the interference suppression is decreasing when $F_{\mathrm{LPF}}$ is increasing, as more intermodulation error is allowed to the control signal. Nevertheless, the delay block is not easy to implement at RF frequency for a wideband operation. Therefore, the main limitation of interference suppression is still the amplitude discrimination between the large interferer and the weak signal.

\section{NIS Operation under Multiple Large Interferers}

In Sections 4 and 5, the working principle of NIS is modeled and analyzed if one local large interferer or one external large interferer is present. In this section, the interference scenario that multiple large interferers, either locally or externally generated, is encountered. The working principle of NIS under such interference scenario is discussed here.

The interference scenario is shown in Figure 24, in which multiple interferers are present. The weak desired signal is shown in red $\left(f_{\text {sig }}\right)$, the local interferers are shown in black and usually are the dominant signal $\left(f_{\text {int1 }}\right.$ and $\left.f_{\text {int2 }}\right)$, and 


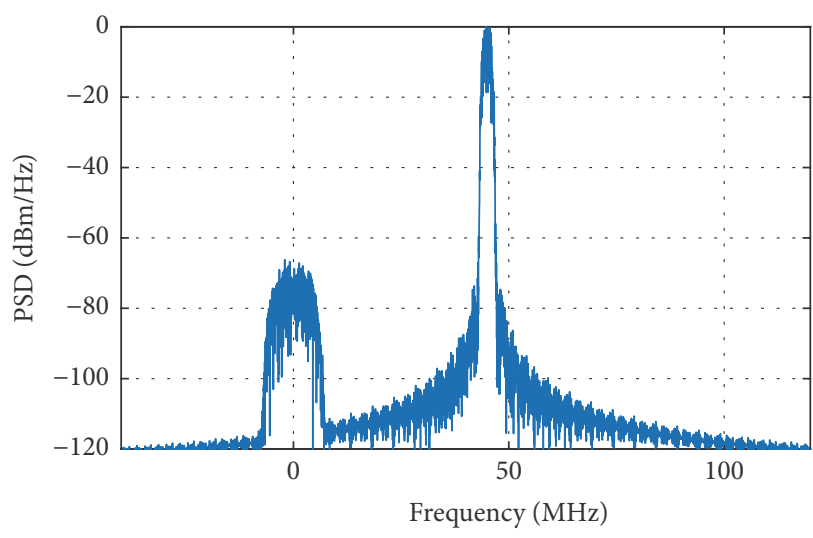

_ NIS input spectrum

(a)

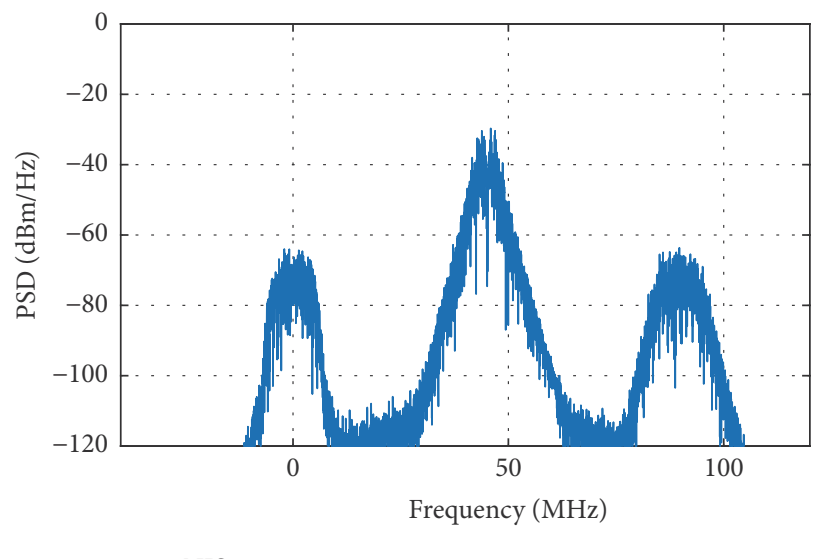

_ NIS output spectrum

(b)

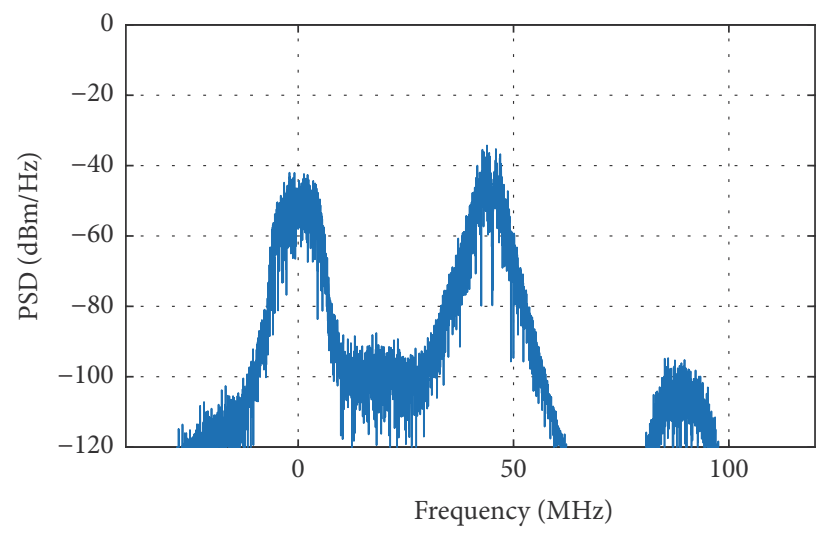

_ NIS baseband spectrum

(c)

FIgURE 21: The frequency spectrum of (a) input at receiver antenna, (b) output of NIS subblock, and (c) baseband output.

TABLE 2: Trade-off of $F_{\text {LPF }}$ on interference suppression.

\begin{tabular}{lcccc}
\hline$F_{\mathrm{LPF}}(\mathrm{MHz})$ & Intermodulation $(\mathrm{dBm})$ & Delay $(\mathrm{ns})$ & Suppression without delay $(\mathrm{dB})$ & Suppression with delay $(\mathrm{dB})$ \\
\hline 5 & -90 & 36 & 30 & 52 \\
10 & -69 & 18 & 38 & 50 \\
20 & -60 & 8 & 46 & 45 \\
\hline
\end{tabular}

external interferers are shown in blue $\left(f_{\text {int3 }}\right.$ and $\left.f_{\text {int } 4}\right)$. The grey line indicates the RF bandwidth of a NIS receiver system. In this case, the worst scenario is that the two large local interferers INT1 and INT2 saturate the receiver, and the 3rd-intermodulation (IM3) product between them is exactly located on the desired signal frequency. Besides, although external interferers INT3 and INT4 are not as powerful as local interferers, they may also saturate the receiver chain.

The NIS operation principle under multiple interferers is illustrated in Figure 25. The local interferers are dominant interferers so they should be filtered out first. Since the local interference envelope is priorly known information, one NIS circuit block can be enabled for suppressing each corresponding local interferer. Therefore the NIS operation prevents the receiver from saturation.
The influence of IM3 product on signal distortion is not alleviated as it happens before the large interferers are suppressed and the NIS operation relies on nonlinear transfer function. On the other hand, for receiver or RF circuit, once $\mathrm{IIP}_{3}$ is known, IM3 at any other power level can be calculated. For every 1-dB increase of the $\mathrm{IIP}_{3}$ point, the corresponding IM3 product drops by $3 \mathrm{~dB}$ [30]. Based on (18), the nonlinear zig-zag transfer function based receiver has an $\operatorname{IIP}_{3} 10 \mathrm{~dB}$ higher than the interferer envelope amplitude $A_{\mathrm{LS}}$, which should help lower the IM3 product amplitude.

Besides, the nonlinear receiver can implement frequencytranslational filtering techniques at mixer and baseband stage, as shown in Figures 5 and 6. Therefore, besides amplitudedomain filtering at the RF stage, the receiver also has frequency-domain filtering at mixer and baseband stage. 


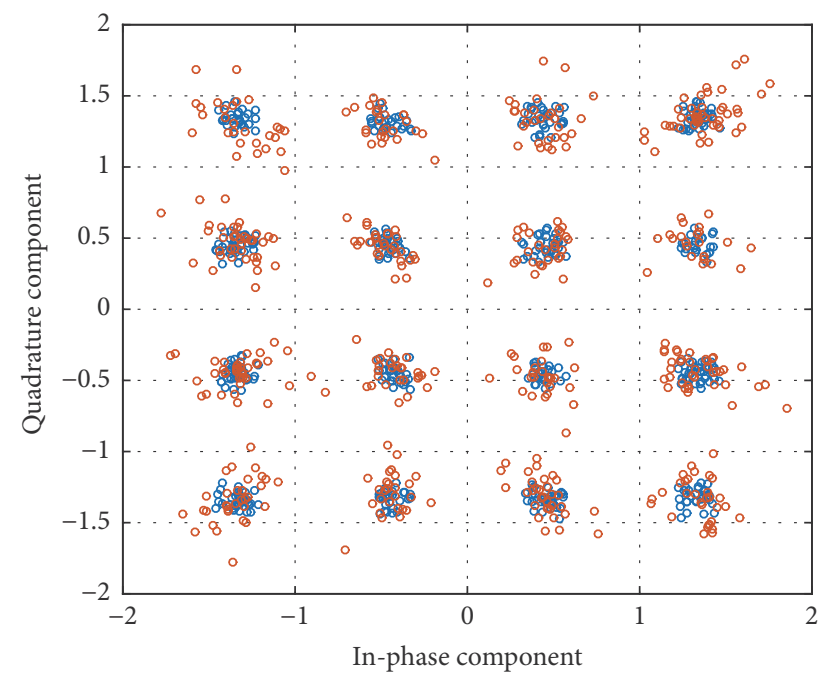

FIGURE 22: Baseband output constellation diagram of the nonlinear receiver system for general large interference suppression.

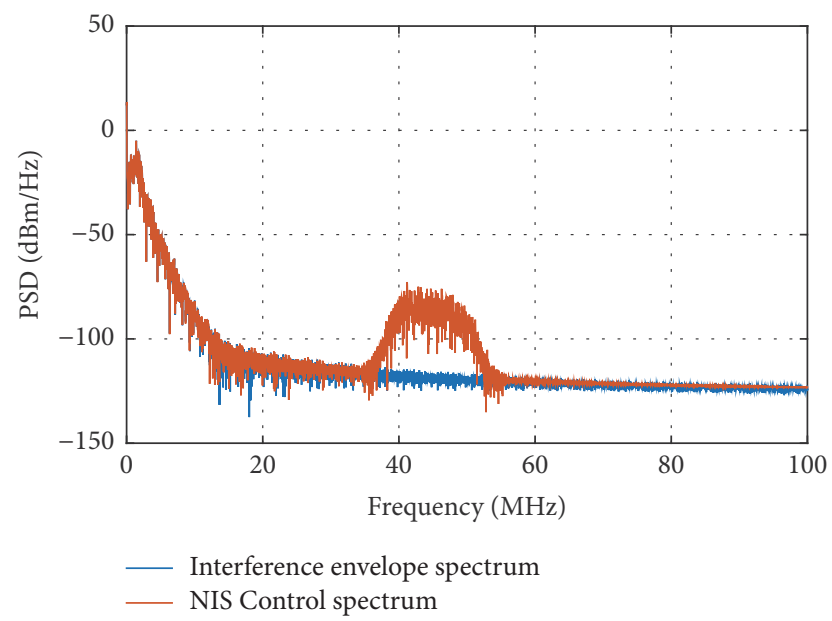

FIGURE 23: The frequency spectrum of envelope of the interferer (blue) and control signal for NIS subblock (red).

The frequency selectivity at the RF input depends on LO frequency sweeping range and baseband filtering order. That helps alleviate the IM3 problem, depending on the frequency spacing between the wanted signal and interferers.

A final NIS circuit block can be enabled if still large external interferers exist. Since the external interferers are usually smaller in power compared with the local ones, it is only necessary to deal with the dominant external interferer. The envelope extraction circuit block will extract the envelope of the dominant external interferer and feed it to the NIS circuit block to partially suppress the external interferer, as discussed in Section 5. In this way, the influences of large interferers are largely alleviated.

\section{Conclusion}

The nonlinear receiver with adaptive nonlinear transfer function has been proposed for multiradio coexistence problems

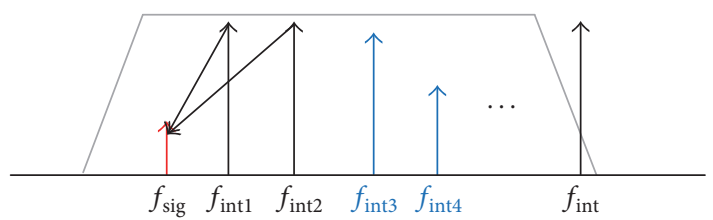

FIGURE 24: Illustration of NIS operation principle with multiple large interferers accompanying weak desired signal (red). The interferers include local interferers (black) and external interferers (blue).

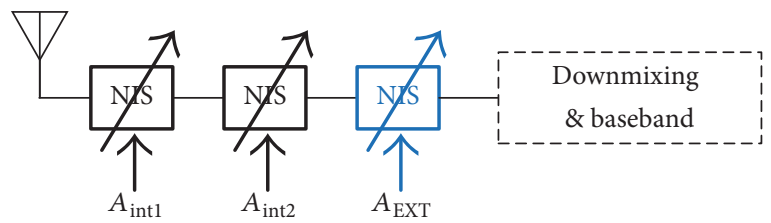

FIGURE 25: Illustration of NIS operation principle with NIS blocks for each local large interferer.

in wideband receivers. It relies on the amplitude information of the interference signal and enables frequency-independent filtering, thus improving in-band and out-of-band linearity for wideband operation. With the nonlinear method, the interference suppression is achieved at the RF stage, which relieves the requirement and power consumption for the following circuitry in the receiver chain. With this method, the interference envelope should be tracked continuously to adjust the nonlinear transfer function accordingly. An adaption method for envelope extraction is proposed and cosimulated with the RF receiver. The limitations for interference suppression are identified. From the analysis, the main limitation of interference suppression is the amplitude discrimination between large and weak signals. In the situation of external interference suppression, the accuracy of the extracted envelope is affected by the LPF filter. Therefore the input frequency separation and bandwidth limit the performance of interference suppression. From system level simulation, a large interference suppression is achieved, and positive SIR can be achieved at the input of baseband ADCs. Therefore the ADC resolution requirement is relaxed and the aliasing product is alleviated.

\section{Competing Interests}

The authors declare that there are no competing interests.

\section{Acknowledgments}

The authors would like to acknowledge the financial contribution of the CORTIF (CA116) project to this work.

\section{References}

[1] ESTI, November 2013, 3rd Generation Partnership Project (3GPP): LTE, http://www.3gpp.org/LTE.

[2] GSMArena, March 2013, http://www.gsmarena.com. 
[3] J. Borremans, B. Van Liempd, E. Martens, S. Cha, and J. Craninckx, "A 0.9V low-power 0.4-6GHz linear SDR receiver in 28nm CMOS," in Proceedings of the Symposium on VLSI Circuits (VLSIC '13), pp. C146-C147, June 2013.

[4] C. Andrews and A. C. Molnar, "A passive mixer-first receiver with digitally controlled and widely tunable RF interface," IEEE Journal of Solid-State Circuits, vol. 45, no. 12, pp. 2696-2708, 2010.

[5] Z. Ru, E. A. M. Klumperink, G. J. M. Wienk, and B. Nauta, "A software-defined radio receiver architecture robust to outof-band interference," in Proceedings of the IEEE International Solid-State Circuits Conference (ISSCC '09), San Francisco, Calif, USA, February 2009.

[6] D. Murphy, A. Hafez, A. Mirzaei et al., "A blocker-tolerant wideband noise-cancelling receiver with a $2 \mathrm{~dB}$ noise figure," in Proceedings of the 59th International Solid-State Circuits Conference (ISSCC '12), pp. 74-75, San Francisco, Calif, USA, February 2012.

[7] D. Murphy, H. Darabi, and H. Xu, "3.6 A noise-cancelling receiver with enhanced resilience to harmonic blockers," in Proceedings of the 61st IEEE International Solid-State Circuits Conference (ISSCC '14), pp. 68-69, IEEE, San Francisco, Calif, USA, February 2014.

[8] H. Wu, M. Mikhemar, D. Murphy, H. Darabi, and M.-C. F. Chang, "A blocker-tolerant inductor-less wideband receiver with phase and thermal noise cancellation," IEEE Journal of Solid-State Circuits, vol. 50, no. 12, pp. 2948-2964, 2015.

[9] F. Lin, P.-I. Mak, and R. P. Martins, "An RF-to-BB-current-reuse wideband receiver with parallel $\mathrm{N}$-path active/passive mixers and a single-MOS pole-zero LPF,' IEEE Journal of Solid-State Circuits, vol. 49, no. 11, pp. 2547-2559, 2014.

[10] Z. Lin, P.-I. Mak, and R. P. Martins, "A $2.4 \mathrm{GHz}$ ZigBee receiver exploiting an RF-to-BB-current-reuse Blixer + hybrid filter topology in $65 \mathrm{~nm}$ CMOS," IEEE Journal of Solid-State Circuits, vol. 49, no. 6, pp. 1333-1344, 2014.

[11] C.-Y. Yu, I. S.-C. Lu, Y.-H. Chen et al., "A SAW-less GSM/GPRS/EDGE receiver embedded in 65-nm SoC", IEEE Journal of Solid-State Circuits, vol. 46, no. 12, pp. 3047-3060, 2011.

[12] Y. Xu, J. Zhu, and P. R. Kinget, "A blocker-tolerant RF front end with harmonic-rejecting N-path filtering," in Proceedings of the IEEE Radio Frequency Integrated Circuits Symposium (RFIC '14), pp. 39-42, Tampa, Fla, USA, June 2014.

[13] E. J. G. Janssen, D. Milosevic, P. G. M. Baltus, A. H. M. Van Roermund, and H. Habibi, "Frequency-independent smart interference suppression for multi-standard transceivers," in Proceedings of the 42nd European Microwave Conference (EuMC '12)-Held as Part of 15th European Microwave Week (EuMW '12), pp. 1289-1292, Amsterdam, The Netherlands, November 2012.

[14] H. Habibi, E. J. G. Janssen, R. G. M. Hilkens et al., "Experimental evaluation of an adaptive nonlinear interference suppressor for multimode transceivers," IEEE Journal on Emerging and Selected Topics in Circuits and Systems, vol. 3, no. 4, pp. 602-614, 2013.

[15] IEEE 802, IEEE 802.11ac Wireless LANs, 2013, http://standards .ieee.org/about/get/802/802.11.html.

[16] SIG, December 2014, Bluetooth Core Specification Version 4.2, https://www.bluetooth.com/specifications.

[17] IEEE 802, December, IEEE 802.16 Wireless LANs, 2008, http:// www.ieee802.org/16/tge/.

[18] LightReading, http://www.lightreading.com.
[19] C. Lu, M. K. Matters-Kammerer, A. Zamanifekri, A. B. Smolders, and P. G. M. Baltus, "A millimeter-wave tunable hybrid-transformer-based circular polarization duplexer with sequentially-rotated antennas," IEEE Transactions on Microwave Theory and Techniques, vol. 64, no. 1, pp. 166-177, 2016.

[20] V. Aparin, G. J. Ballantyne, C. J. Persico, and A. Cicalini, "An integrated LMS adaptive filter of TX leakage for CDMA receiver front ends," IEEE Journal of Solid-State Circuits, vol. 41, no. 5, pp. 1171-1182, 2006.

[21] A. Mirzaie, A. Yazdi, Z. Zhou, E. Chang, P. Suri, and H. Darabi, "A $65 \mathrm{~nm}$ CMOS quad-band SAW-less receiver for GSM/GPRS/EDGE," in Proceedings of the 24th Symposium on VLSI Circuits (VLSIC '10), pp. 179-180, IEEE, Honolulu, Hawaii, USA, June 2010.

[22] H. Darabi, "A blocker filtering technique for SAW-less wireless receivers," IEEE Journal of Solid-State Circuits, vol. 42, no. 12, pp. 2766-2773, 2007.

[23] S. Youssef, R. van der Zee, and B. Nauta, "Active feedback technique for RF channel selection in front-end receivers," IEEE Journal of Solid-State Circuits, vol. 47, no. 12, pp. 3130-3144, 2012.

[24] D. Arnstein, T. Czerner, and J. Buzzelli, "Broadband signal processing for AJ and RFI reduction in spread spectrum systems," in Proceedings of the Military Communications Conference (MILCOM '94), vol. 2, pp. 421-429, IEEE, Fort Monmouth, NJ, USA, 1994, Conference Record.

[25] H. Habibi, E. J. G. Janssen, Y. Wu, D. Milosevic, J. W. M. Bergmans, and P. G. M. Baltus, "Suppression of constant modulus interference in multimode transceivers using an adaptive nonlinear circuit," in Proceedings of the NASA/ESA Conference on Adaptive Hardware and Systems (AHS '13), pp. 150-155, IEEE, Torino, Italy, June 2013.

[26] E. J. G. Janssen, D. Milosevic, and P. G. M. Baltus, "A $1.8 \mathrm{GHz}$ amplifier with $39 \mathrm{~dB}$ frequency-independent smart self-interference blocker suppression," in Proceedings of the IEEE Radio Frequency Integrated Circuits Symposium (RFIC '12), pp. 97-100, IEEE, Montreal, Canada, June 2012.

[27] J. Steward, Calculus: Early Transcendentals, Brooks Cole, 2003.

[28] N. M. Blachman, "Bandpass nonlinearities," IEEE Transactions on Information Theory, vol. 10, no. 2, pp. 162-164, 1964.

[29] H. Habibi, E. J. G. Janssen, W. Yan, J. W. M. Bergmans, and P. G. M. Baltus, "System study on nonlinear suppression of varying-envelope local interference in multimode transceivers," International Journal of Electronics and Communications, vol. 69, no. 7, pp. 963-973, 2015.

[30] B. Razavi, RF Microelectronic, Pearson Education, 2nd edition, 2012. 


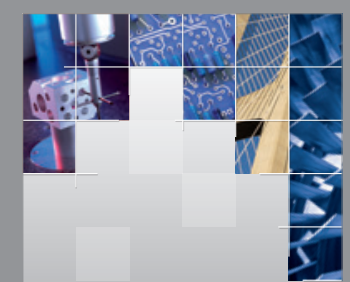

\section{Enfincering}
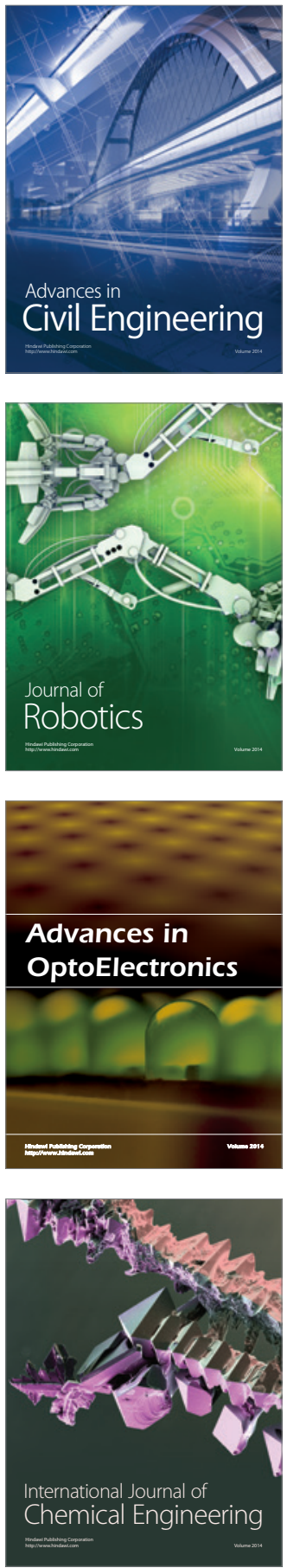

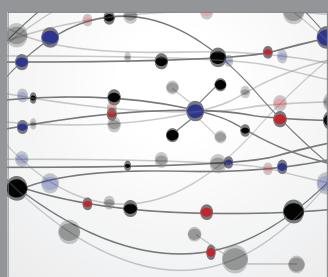

The Scientific World Journal

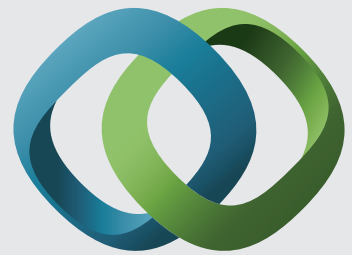

\section{Hindawi}

Submit your manuscripts at

https://www.hindawi.com
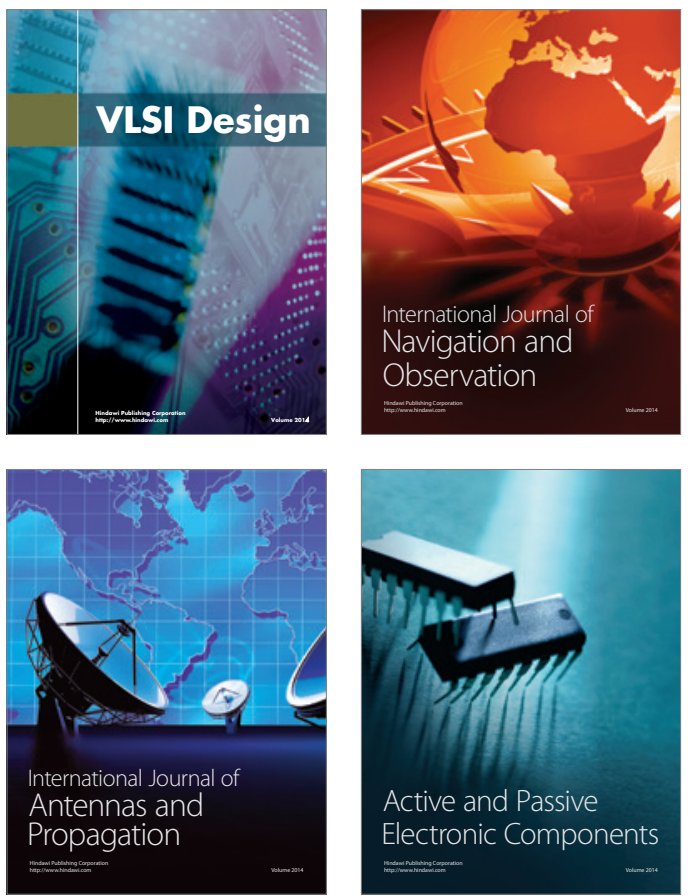
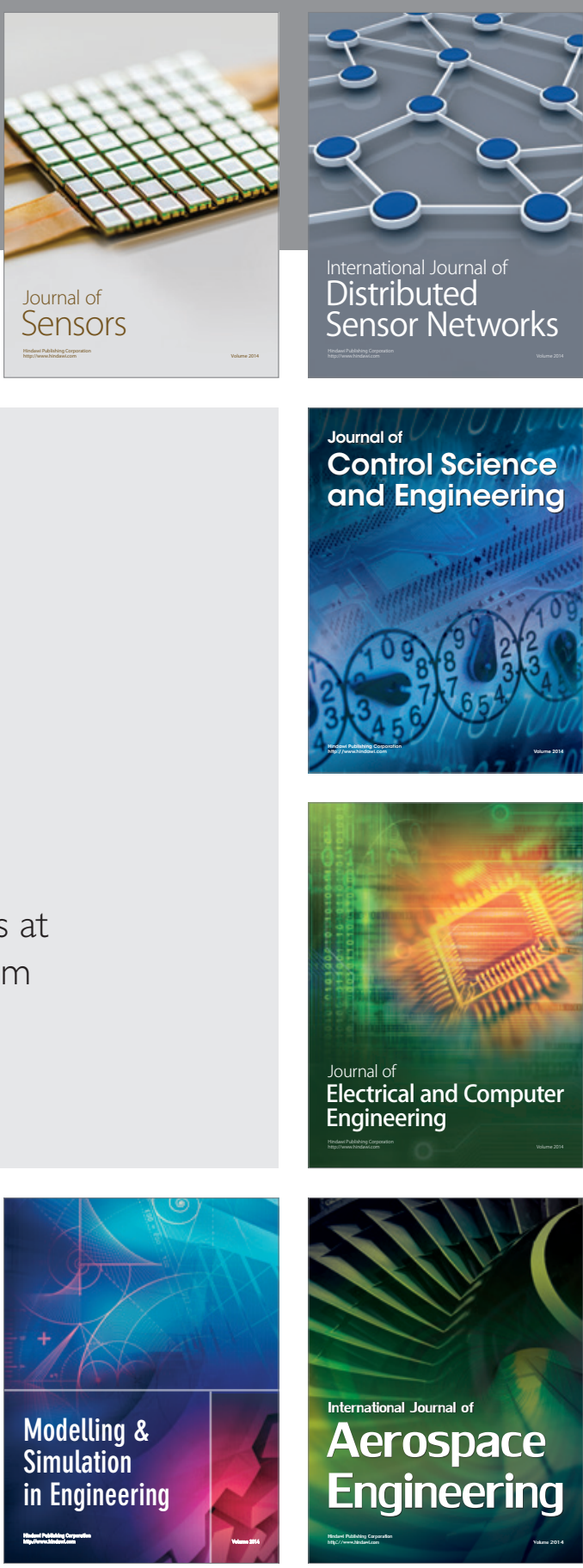

International Journal of

Distributed

Sensor Networks

$-$

Joumal of

Control Science

and Engineering
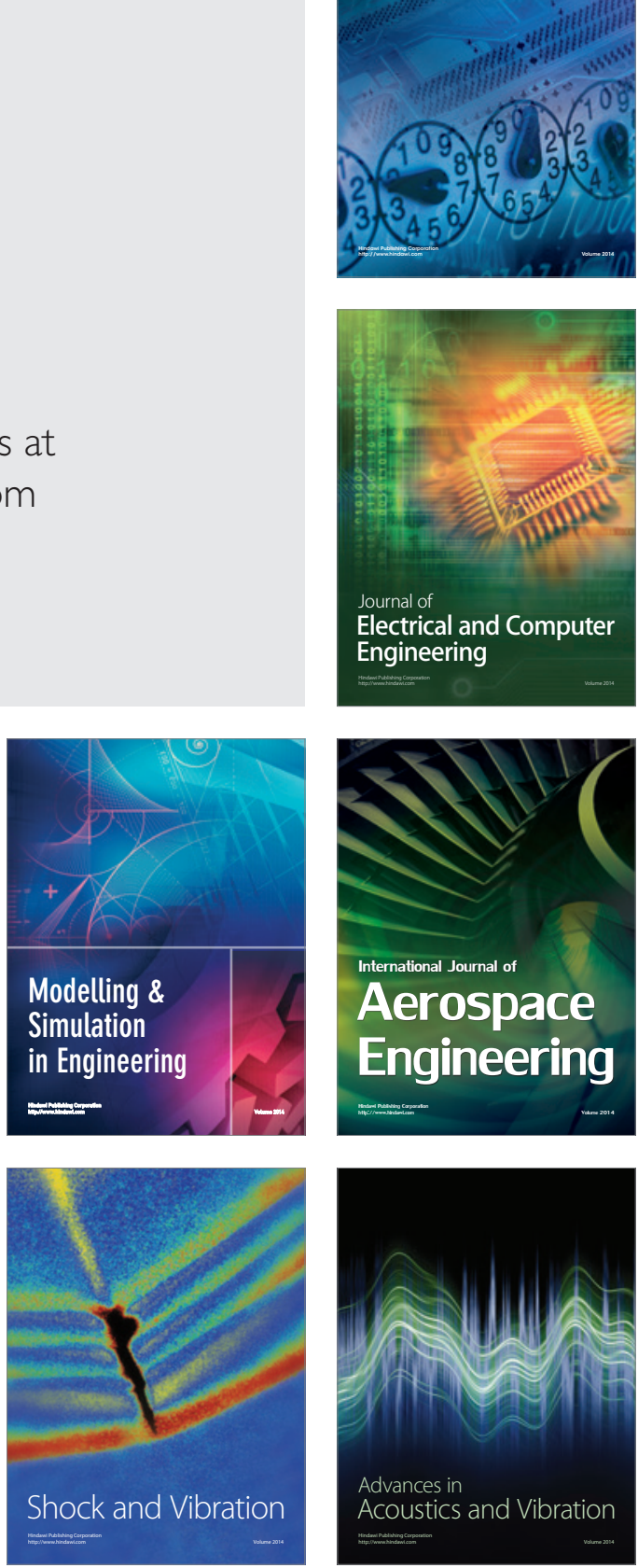